\title{
AN OPTIMALLY ACCURATE DISCRETE REGULARIZATION FOR SECOND ORDER TIMESTEPPING METHODS FOR NAVIER-STOKES EQUATIONS
}

\author{
NAN JIANG*, MUHAMMAD MOHEBUJJAMAN ${ }^{\dagger}$, LEO G. REBHOLZ ${ }^{\ddagger}$, AND CATALIN \\ TRENCHEA $\S$
}

\begin{abstract}
We propose a new, optimally accurate numerical regularization/stabilization for (a family of) second order timestepping methods for the Navier-Stokes equations (NSE). The method combines a linear treatment of the advection term, together with a stabilization terms that are proportional to discrete curvature of the solutions in both velocity and pressure. We rigorously prove that the entire new family of methods are unconditionally stable and $O\left(\Delta t^{2}\right)$ accurate. The idea of 'curvature stabilization' is new to CFD and is intended as an improvement over the commonly used 'speed stabilization', which is only first order accurate in time and can have an adverse affect on important flow quantities such as drag coefficients. Numerical examples verify the predicted convergence rate and show the stabilization term clearly improves the stability and accuracy of the tested flows.
\end{abstract}

Key words. Navier-Stokes, Unconditional stability, IMEX methods, second order convergence, Crank-Nicolson, BDF2

AMS subject classifications. 76D05, 65L20, 65M12

1. Introduction. We consider optimally accurate stabilizations for second order time-stepping methods for the Navier-Stokes equations (NSE) on a bounded domain $\Omega \subseteq \mathbb{R}^{d}, \mathrm{~d}=2$ or 3 :

$$
\begin{aligned}
\mathbf{u}_{t}+\mathbf{u} \cdot \nabla \mathbf{u}-\nu \triangle \mathbf{u}+\nabla p & =\mathbf{f}, \text { for } x \in \Omega, 0<t \leq T, \\
\nabla \cdot \mathbf{u} & =0, \text { for } x \in \Omega, 0<t \leq T, \\
\mathbf{u} & =0, \text { on } \partial \Omega, \text { for } 0<t \leq T, \\
\mathbf{u}(x, 0) & =\mathbf{u}_{0}(x), \text { for } x \in \Omega,
\end{aligned}
$$

with the pressure satisfying the usual zero-mean normalization.

Developing efficient, accurate, and robust numerical methods for solving the NSE remains a great challenge in Computational Fluid Dynamics (CFD). For time-stepping methods, common approaches combine linearizations at each timestep with stabilizations/regularizations that damp oscillations and unstable modes. An important linearization method is CNLE (Crank-Nicolson with linear extrapolation), proposed by Baker [2], which is comparable in stability and accuracy with the more expensive, fully implicit Crank-Nicolson (CN) method, [31, 1, 14]. However, while a nonlinear solver for $\mathrm{CN}$ requires several linear solves at each time step, CNLE requires just one. A similar linearization exists for BDF2 timestepping (called BDF2LE). Herein, we will consider a new, optimally accurate stabilization to be used with CNLE, BDF2LE,

*Department of Scientific Computing, Florida State University, Tallahassee, FL 32312, Email: njiang@fsu.edu. Partially supported by Air Force grant FA 9550-12-1-0191.

${ }^{\dagger}$ Department of Mathematical Sciences, Clemson University, Clemson SC 29634, Email: mmohebu@clemson.edu. Partially supported by NSF Grant DMS15222191.

‡Department of Mathematical Sciences, Clemson University, Clemson SC 29634, Email: rebholz@clemson.edu. Partially supported by NSF Grant DMS1522191 and US Army Grant 65294MA.

$\S$ Department of Mathematics, 301 Thackeray Hall, University of Pittsburgh, Pittsburgh, PA 15260, Email: trenchea@pitt.edu. Partially supported by Air Force grant FA 9550-12-1-0191 and NSF grant DMS-1522574. 
and the family of methods 'in between' them. Recall this family of methods (without stabilization) is given by

$$
\begin{aligned}
\frac{\left(\theta+\frac{1}{2}\right) \mathbf{u}_{n+1}-2 \theta \mathbf{u}_{n}+\left(\theta-\frac{1}{2}\right) \mathbf{u}_{n-1}}{\triangle t}-\theta \nu \triangle \mathbf{u}_{n+1}-\nu(1-\theta) \triangle \mathbf{u}_{n} & \\
+\left((\theta+1) \mathbf{u}_{n}-\theta \mathbf{u}_{n-1}\right) \cdot \nabla\left(\theta \mathbf{u}_{n+1}+(1-\theta) \mathbf{u}_{n}\right)+\theta \nabla p_{n+1}+(1-\theta) \nabla p_{n} & =\mathbf{f}_{n+\theta}, \\
\nabla \cdot \mathbf{u}_{n+1} & =0, \quad(1.3)
\end{aligned}
$$

where $\theta \in\left[\frac{1}{2}, 1\right]$. If $\theta=1$, BDF2LE is recovered, and if $\theta=\frac{1}{2}$, then CNLE is recovered. For any other $\theta \in\left(\frac{1}{2}, 0\right)$, a second order method is still recovered. Since CNLE exactly conserves energy, and BDF2LE numerically dissipates it, the parameter $\theta$ can be used to control the dissipation.

A successful stabilization method to be used with (1.2) must be able to damp the instabilities that frequently arise in NSE simulations, but without over-smoothing or removing important flow structures, i.e. without hurting accuracy. A common approach for these timestepping methods is to add $-\alpha \Delta \mathbf{u}_{n+1}$ to the left hand side, and $-\alpha \Delta \mathbf{u}_{n}$ to the right hand side, where $\alpha$ is a tuning parameter generally taken to be on the order of the meshwidth $h$. Such a stabilization has been used in methods for Navier-Stokes (see [23, 8] and references therein), and is related in principle to techniques used in turbulence modeling [20,17], ocean modeling [5], and also to the discretization of the 'Voigt term' in a turbulence model recently studied by Titi and others, e.g. [6, 22]. As shown in these works, this stabilization can be effective for several different types of flows, and also can improve conditioning of linear systems by increasing the coefficient of the stiffness matrix, e.g. in the case of BDF2LE, from $\nu$ to $\nu+\alpha$. However, as shown in the analysis of [23], this technique is $O(\alpha \Delta t)$ accurate, and thus can potentially be a dominant error source in second order timestepping methods if the usual choice of $\alpha=O(h)$ is made. If one instead takes $\alpha=O(\Delta t)$, this creates a need for a careful retuning of $\alpha$ each time the time step size is changed, which could make its use with adaptive time-stepping very difficult.

The purpose of this paper is to introduce and analyze a new stabilization for timestepping methods of the form (1.2), that can sufficiently stabilize, but is $O\left(\Delta t^{2}\right)$ accurate, which is optimally accurate for second order timestepping methods. The design of the stabilization is inspired by the idea of stabilizing 'curvature' $\left(\mathbf{u}_{n+1}-2 \mathbf{u}_{n}+\mathbf{u}_{n-1}\right)$, instead of stabilizing 'speed' $\left(\mathbf{u}_{n+1}-\mathbf{u}_{n}\right)$, which is done by the stabilization discussed above. Not only is curvature stabilization more accurate than speed stabilization (with respect to $\Delta t$ ), but in CFD it does not directly alter important flow quantities such as drag coefficients, as speed penalization does (see section 5.3). To our knowledge, the idea of curvature stabilization is new to CFD, and was first introduced very recently $[32,33]$ as a timestepping method for two particular classes of ODEs. We note that an interface stabilization term for a Stokes-Darcy system in the recent paper [7] could also be considered to be in the same spirit, although their interpretation was somewhat different, and their error analysis led to a similar second order curvature-type term. In the numerical weather and climate prediction models, the Robert-Asselin time filter and its refinements (the Robert-Asselin-Williams [35], the higher-order Robert-Asselin time filter [27], etc.) have a similar stabilizing 'curvature' effect (see e.g. [18, 26]).

The new family of second-order, unconditionally stable, IMEX time-stepping 
methods we propose are given by:

$$
\begin{aligned}
& \frac{\left(\theta+\frac{1}{2}\right) \mathbf{u}_{n+1}-2 \theta \mathbf{u}_{n}+\left(\theta-\frac{1}{2}\right) \mathbf{u}_{n-1}}{\triangle t} \\
& -\theta(\nu+\epsilon) \triangle \mathbf{u}_{n+1}-(\nu-\theta(\nu+2 \epsilon)) \triangle \mathbf{u}_{n}-\theta \epsilon \triangle \mathbf{u}_{n-1} \\
& +\left((\theta+1) \mathbf{u}_{n}-\theta \mathbf{u}_{n-1}\right) \cdot \nabla\left(\theta \frac{\nu+\epsilon}{\nu} \mathbf{u}_{n+1}+\left(1-\theta \frac{\nu+2 \epsilon}{\nu}\right) \mathbf{u}_{n}+\theta \frac{\epsilon}{\nu} \mathbf{u}_{n-1}\right) \\
& +\theta \frac{\nu+\epsilon}{\nu} \nabla p_{n+1}+\left(1-\theta \frac{\nu+2 \epsilon}{\nu}\right) \nabla p_{n}+\theta \frac{\epsilon}{\nu} \nabla p_{n-1}=\mathbf{f}_{n+\theta}, \\
& \nabla \cdot \mathbf{u}_{n+1}=0,
\end{aligned}
$$

where $\theta \in\left[\frac{1}{2}, 1\right], \epsilon \geq 0$. Our analysis will show there is optimal convergence for any constant $\epsilon$, however the natural norms of the problem dictate that $\epsilon=O(\nu)$ is most appropriate for simulations. Although this appears to be quite small compared to stabilization constants in other common methods, our numerical experiments demonstrate that such $\epsilon$ do provide sufficient stabilization.

The family of methods (1.4) are stabilizations of the family of methods (1.2), and if $\epsilon=0$, then (1.2) is recovered. Thus, for $\theta=1$ in (1.4), we obtain a regularized BDF2LE scheme which we will call BDF2LEREG. Similarly, the scheme (1.4) with $\theta=\frac{1}{2}$ will be called CNLEREG. Our stability and convergence analysis will be general, and apply to the entire family (1.4); we prove both unconditional stability and $O\left(\Delta t^{2}\right)$ accuracy. For our numerical examples, we perform numerical tests with both CNLEREG and BDF2LEREG. As is typical with stabilization methods, the positive effect is seen more through numerical tests than through analysis, and we provide several examples which show convincing evidence of the effectiveness of the proposed stabilization.

The analysis we perform herein to prove unconditional stability and optimal convergence can be applied to other advection-diffusion models, e.g., Lions' hyperviscosity model [28], the modified NSE of Ladyzhenskaya [24] and nonlinear spectral eddyviscosity models of turbulence [11]. In particular, we note our proposed method(s) are related to one discussed by H. Johnston and J.G. Liu in [21], and our analysis can be extended to show that (3.17) in [21] is stable (it is stated as an open problem in the paper). We note also that our analysis also applies in a straightforward manner to the implicit version of (1.4).

This paper is arranged as follows. In section 2, we give notation and preliminaries to make for a smoother analysis to follow. Section 3 proves unconditional stability of the proposed family of methods, and section 4 proves optimal convergence for them. In section 5, we provide numerical tests that show the methods are very effective on several benchmark problems. Finally, conclusions are drawn in section 6 .

2. Notations and Preliminaries. Let $\Omega$ be an open, regular domain in $\mathbb{R}^{d}$, $d=2$ or 3 . We denote the usual $L^{2}(\Omega)$ norm and the inner product by $\|\cdot\|$ and $(\cdot, \cdot)$. The $L^{p}(\Omega)$ norms and the Sobolev $W_{p}^{k}(\Omega)$ norms are denoted by $\|\cdot\|_{L^{p}}$ and $\|\cdot\|_{W_{p}^{k}}$ respectively. In particular, $H^{k}(\Omega)$ is used to represent the Sobolev space $W_{2}^{k}(\Omega)$. $\|\cdot\|_{k}$ and $|\cdot|_{k}$ denote the norm and the seminorm in $H^{k}(\Omega)$. For functions $v(x, t)$ defined on the time interval $(0, T)$, we define $(1 \leq m<\infty)$

$$
\|v\|_{\infty, k}:=\operatorname{EssSup}_{[0, T]}\|v(t, \cdot)\|_{k} \quad \text { and } \quad\|v\|_{m, k}:=\left(\int_{0}^{T}\|v(t, \cdot)\|_{k}^{m} d t\right)^{1 / m}
$$


We consider the finite element method (FEM) for the spatial discretization. Let $X$ be the velocity space and $Q$ be the pressure space:

$$
\begin{aligned}
X & :=\left(H_{0}^{1}(\Omega)\right)^{d}=\left\{\mathbf{v}: \mathbf{v} \in\left(H^{1}(\Omega)\right)^{d}, \mathbf{v}=0 \text { on } \partial \Omega\right\} \\
Q & :=L_{0}^{2}(\Omega)=\left\{q: q \in L^{2}(\Omega), \int_{\Omega} q d x=0\right\}
\end{aligned}
$$

A weak formulation of (1.1) is: Find $\mathbf{u}:[0, T] \rightarrow X, p:[0, T] \rightarrow Q$ for a.e. $t \in(0, T]$ satisfying

$$
\begin{aligned}
\left(\mathbf{u}_{t}, \mathbf{v}\right)+(\mathbf{u} \cdot \nabla \mathbf{u}, \mathbf{v})+\nu(\nabla \mathbf{u}, \nabla \mathbf{v})-(p, \nabla \cdot \mathbf{v}) & =(\mathbf{f}, \mathbf{v}), \forall \mathbf{v} \in X \\
\mathbf{u}(x, 0)=\mathbf{u}^{0}(x) \text { in } X \text { and }(\nabla \cdot \mathbf{u}, q) & =0, \forall q \in Q
\end{aligned}
$$

The space of divergence free functions is given by

$$
V:=\{\mathbf{v} \in X:(\nabla \cdot \mathbf{v}, q)=0, \forall q \in Q\} .
$$

Let $V^{*}$ denote the dual space of $V$. The norm $\|\cdot\|_{*}$ on $V^{*}$ is defined as

$$
\|\mathbf{f}\|_{*}:=\sup _{0 \neq \mathbf{v} \in V} \frac{(\mathbf{f}, \mathbf{v})}{\|\nabla \mathbf{v}\|} .
$$

Let $X^{h} \subset X, Q^{h} \subset Q$ denote conforming velocity, pressure finite element spaces based on an edge to edge triangulations of $\Omega$ with maximum triangle diameter $h$. The velocity-pressure FEM spaces $\left(X^{h}, Q^{h}\right)$ are assumed to satisfy the usual discrete inf-sup / $L B B^{h}$ condition, see Gunzburger's book [12], for stability of the discrete pressure:

$$
\inf _{q^{h} \in Q^{h}} \sup _{\mathbf{v}^{h} \in X^{h}} \frac{\left(q^{h}, \nabla \cdot \mathbf{v}^{h}\right)}{\left\|q^{h}\right\|\left\|\nabla \mathbf{v}^{h}\right\|} \geq \beta>0
$$

where $\beta$ is independent of $h$. Taylor-Hood elements, [12], are one commonly used choice of such velocity-pressure finite element spaces. Other choices can also be found in $[12,9]$. We also assume the following approximation properties typical of piecewise polynomials of degree $(k, k-1)$, see [4], hold for $\left(X^{h}, Q^{h}\right)$ :

$$
\begin{aligned}
& \inf _{\mathbf{v}^{h} \in X^{h}}\left\|\mathbf{u}-\mathbf{v}^{h}\right\| \leq C h^{k+1}|\mathbf{u}|_{k+1}, \mathbf{u} \in H^{k+1}(\Omega), \\
& \inf _{\mathbf{v}^{h} \in X^{h}}\left\|\nabla\left(\mathbf{u}-\mathbf{v}^{h}\right)\right\| \leq C h^{k}|\mathbf{u}|_{k+1}, \mathbf{u} \in H^{k+1}(\Omega), \\
& \inf _{q^{h} \in Q^{h}}\left\|p-q^{h}\right\| \leq C h^{k}|p|_{k}, p \in H^{k}(\Omega) .
\end{aligned}
$$

The discretely divergence free subspace of $X^{h}$ is

$$
V^{h}:=\left\{\mathbf{v}^{h} \in X^{h}:\left(\nabla \cdot \mathbf{v}^{h}, q^{h}\right)=0, \forall q^{h} \in Q^{h}\right\} .
$$

Define the usual explicitly skew symmetrized trilinear form

$$
b^{*}(\mathbf{u}, \mathbf{v}, \mathbf{w}):=\frac{1}{2}(\mathbf{u} \cdot \nabla \mathbf{v}, \mathbf{w})-\frac{1}{2}(\mathbf{u} \cdot \nabla \mathbf{w}, \mathbf{v}) .
$$

$b^{*}(\mathbf{u}, \mathbf{v}, \mathbf{w})$ satisfies the bound, see [9],

$$
\left|b^{*}(\mathbf{u}, \mathbf{v}, \mathbf{w})\right| \leq C(\Omega)\|\nabla \mathbf{u}\|\|\nabla \mathbf{v}\|\|\nabla \mathbf{w}\|, \forall \mathbf{u}, \mathbf{v}, \mathbf{w} \in X,
$$


and, if $\mathbf{v}, \nabla \mathbf{v} \in L^{\infty}(\Omega)$ (see e.g., Lemma 2.1 in [23]),

$$
\left|b^{*}(\mathbf{u}, \mathbf{v}, \mathbf{w})\right| \leq C(\Omega)\left(\|\mathbf{v}\|_{L^{\infty}(\Omega)}+\|\nabla \mathbf{v}\|_{L^{\infty}(\Omega)}\right)\|\mathbf{u}\|\|\nabla \mathbf{w}\| .
$$

To simplify the analysis, we denote the interpolation at $t=t_{n+\theta}$ by

$$
\mathcal{J}_{n+\theta}^{\epsilon}(a):=\theta \frac{\nu+\epsilon}{\nu} a_{n+1}+\left(1-\theta \frac{\nu+2 \epsilon}{\nu}\right) a_{n}+\theta \frac{\epsilon}{\nu} a_{n-1},
$$

and extrapolation at $t=t_{n+\theta}$ by

$$
\mathcal{H}_{n+\theta}(a):=(\theta+1) a_{n}-\theta a_{n-1} .
$$

The fully discrete approximation of (1.1) we study is: Given $\mathbf{u}_{n}^{h}, \mathbf{u}_{n-1}^{h}, p_{n}^{h}, p_{n-1}^{h}$, find $\mathbf{u}_{n+1}^{h} \in X^{h}, p_{n+1}^{h} \in Q^{h}$ satisfying

$$
\begin{aligned}
& \left(\frac{\left(\theta+\frac{1}{2}\right) \mathbf{u}_{n+1}^{h}-2 \theta \mathbf{u}_{n}^{h}+\left(\theta-\frac{1}{2}\right) \mathbf{u}_{n-1}^{h}}{\triangle t}, \mathbf{v}^{h}\right)+\nu\left(\mathcal{J}_{n+\theta}^{\epsilon}\left(\nabla \mathbf{u}^{h}\right), \nabla \mathbf{v}^{h}\right) \\
& \quad+b^{*}\left(\mathcal{H}_{n+\theta}\left(\mathbf{u}^{h}\right), \mathcal{J}_{n+\theta}^{\epsilon}\left(\mathbf{u}^{h}\right), \mathbf{v}^{h}\right)-\left(\mathcal{J}_{n+\theta}^{\epsilon}\left(p^{h}\right), \nabla \cdot \mathbf{v}^{h}\right)=\left(\mathbf{f}_{n+\theta}, \mathbf{v}^{h}\right), \forall \mathbf{v}^{h} \in X^{h}, \\
& \left(\nabla \cdot \mathbf{u}_{n+1}^{h}, q^{h}\right)=0, \forall q^{h} \in Q^{h} .
\end{aligned}
$$

3. Stability Analysis. We prove the unconditional long time stability of (2.1) using a generalization of the G-stability of the 2nd order backward differentiation method (see for instance the classical book by Hairer and Wanner [13, page 332]; also [32, page 4] and [34, page 4]). To analyze the stability, we first introduce two symmetric matrices. More specifically, we define the symmetric positive matrix $F \in$ $\mathbb{R}^{n \times n}$ by

$$
F=\theta(2 \theta-1) I+\frac{4 \theta^{4} \epsilon}{\nu} I
$$

and the symmetric matrix $G \in \mathbb{R}^{2 n \times 2 n}$ as follows

$$
G=\left(\begin{array}{cc}
\frac{\theta(2 \theta+3)}{4} \frac{\nu+\epsilon}{\nu} I-\frac{\theta(2 \theta+1)}{4} \frac{\epsilon}{\nu} I & -\left(\frac{(\theta+1)(2 \theta-1)}{4} \frac{\nu+\epsilon}{\nu} I+\frac{(1-\theta)(2 \theta+1)}{4} \frac{\epsilon}{\nu} I\right) \\
-\left(\frac{(\theta+1)(2 \theta-1)}{4} \frac{\nu+\epsilon}{\nu} I+\frac{(1-\theta)(2 \theta+1)}{4} \frac{\epsilon}{\nu} I\right) & \frac{\theta(2 \theta-1)}{4} \frac{\nu+\epsilon}{\nu} I+\frac{\theta(-2 \theta+3)}{4} \frac{\epsilon}{\nu} I
\end{array}\right) .
$$

For any $\mathbf{u}, \mathbf{v} \in \mathbb{R}^{n}$, define $F$-norm of the $n$ vector $\mathbf{u}$ :

$$
\|\mathbf{u}\|_{F}=(\mathbf{u}, F \mathbf{u})
$$

which is non-negative, and $G-$ norm of the $2 n$ vector $\left[\begin{array}{l}\mathbf{u} \\ \mathbf{v}\end{array}\right]$ :

$$
\left\|\left[\begin{array}{l}
\mathbf{u} \\
\mathbf{v}
\end{array}\right]\right\|_{G}^{2}=\left(\left[\begin{array}{l}
\mathbf{u} \\
\mathbf{v}
\end{array}\right], G\left[\begin{array}{l}
\mathbf{u} \\
\mathbf{v}
\end{array}\right]\right)
$$

whose value could be negative.

LEMMA 3.1. For any vector $\mathbf{u}, \mathbf{v} \in \mathbb{R}^{n}$, we have

$$
\begin{aligned}
\left(\left[\begin{array}{l}
\mathbf{u} \\
\mathbf{v}
\end{array}\right], G\left[\begin{array}{l}
\mathbf{u} \\
\mathbf{v}
\end{array}\right]\right)= & \frac{2 \theta+1}{4}\|\mathbf{u}\|^{2}+\frac{-2 \theta+1}{4}\|\mathbf{v}\|^{2} \\
& +\frac{(\theta+1)(2 \theta-1)}{4}\|\mathbf{u}-\mathbf{v}\|^{2}+\frac{\theta}{2} \frac{\epsilon}{\nu}\|\mathbf{u}-\mathbf{v}\|^{2} \\
\geq & \frac{2 \theta+1}{4}\|\mathbf{u}\|^{2}-\frac{2 \theta-1}{4}\|\mathbf{v}\|^{2},
\end{aligned}
$$


and

$$
\begin{aligned}
& \left(\left[\begin{array}{l}
\mathbf{u} \\
\mathbf{v}
\end{array}\right], G\left[\begin{array}{l}
\mathbf{u} \\
\mathbf{v}
\end{array}\right]\right) \leq \frac{2 \theta+1}{4}\|\mathbf{u}\|^{2}+\frac{(\theta+1)(2 \theta-1)}{4}\|\mathbf{u}-\mathbf{v}\|^{2}+\frac{\theta}{2} \frac{\epsilon}{\nu}\|\mathbf{u}-\mathbf{v}\|^{2} \\
\leq & \left(\frac{2 \theta+1}{4}+\frac{(\theta+1)(2 \theta-1)}{2}+\frac{\theta \epsilon}{\nu}\right)\|\mathbf{u}\|^{2}+\left(\frac{(\theta+1)(2 \theta-1)}{2}+\frac{\theta \epsilon}{\nu}\right)\|\mathbf{v}\|^{2} .
\end{aligned}
$$

Proof. The equality in (3.1) is obtained by direct algebraic manipulations. As $\theta \in\left[\frac{1}{2}, 1\right]$, we have $(\theta+1)(2 \theta-1) \geq 0$ and thus the inequality in (3.1) follows after deleting the non-negative terms.

Similarly, with $\theta \in\left[\frac{1}{2}, 1\right]$ we have

$$
\frac{-2 \theta+1}{4}\|v\|^{2}=-\frac{\theta-\frac{1}{2}}{2}\|v\|^{2} \leq 0 .
$$

Using the inequality in (3.1) and deleting the above non-positive term gives the first inequality in (3.2). Moreover, as $\|u-v\|^{2} \leq 2\left(\|u\|^{2}+\|v\|^{2}\right)$, the second inequality in (3.2) follows directly.

THEOREM 3.2. The algorithm (2.1) is unconditionally stable and $\forall N \geq 1$, the following energy estimate holds

$$
\begin{gathered}
\left\|\mathbf{u}_{N}^{h}\right\|^{2}+\frac{1}{2 \theta+1} \sum_{n=1}^{N-1}\left\|\mathbf{u}_{n+1}^{h}-2 \mathbf{u}_{n}^{h}+\mathbf{u}_{n-1}^{h}\right\|_{F}^{2}+\frac{2 \triangle t \nu}{2 \theta+1} \sum_{n=1}^{N-1}\left\|\mathcal{J}_{n+\theta}^{\epsilon}\left(\nabla \mathbf{u}^{h}\right)\right\|^{2} \\
\leq\left(\frac{2 \theta-1}{2 \theta+1}\right)^{N}\left\|\mathbf{u}_{0}^{h}\right\|^{2}+\frac{4 N}{2 \theta+1}\left\|\left[\begin{array}{c}
\mathbf{u}_{1}^{h} \\
\mathbf{u}_{0}^{h}
\end{array}\right]\right\|_{G}^{2}+\frac{2 N \triangle t}{\nu(2 \theta+1)} \sum_{n=1}^{N-1}\left\|\mathbf{f}_{n+\theta}\right\|_{*}^{2} .
\end{gathered}
$$

REMARK 3.1. Setting $\theta=1$ in the stability result above and expanding the term $\mathcal{J}_{n+\theta}^{\epsilon}\left(\nabla \mathbf{u}^{h}\right)$ gives the BDF2LEREG stability result,

$$
\begin{aligned}
\left\|\mathbf{u}_{N}^{h}\right\|^{2} & +\frac{1}{3} \sum_{n=1}^{N-1}\left\|\mathbf{u}_{n+1}^{h}-2 \mathbf{u}_{n}^{h}+\mathbf{u}_{n-1}^{h}\right\|_{F}^{2} \\
& +\frac{2 \triangle t \nu}{3} \sum_{n=1}^{N-1}\left\|\nabla \mathbf{u}_{n+1}^{h}+\frac{\epsilon}{\nu} \nabla\left(\mathbf{u}_{n+1}^{h}-2 \mathbf{u}_{n}^{h}+\mathbf{u}_{n-1}^{h}\right)\right\|^{2} \\
& \leq\left(\frac{1}{3}\right)^{N}\left\|\mathbf{u}_{0}^{h}\right\|^{2}+\frac{4 N}{3}\left\|\left[\begin{array}{c}
\mathbf{u}_{1}^{h} \\
\mathbf{u}_{0}^{h}
\end{array}\right]\right\|_{G}^{2}+\frac{2 N \triangle t}{3 \nu} \sum_{n=1}^{N-1}\left\|\mathbf{f}_{n+1}\right\|_{*}^{2} .
\end{aligned}
$$

We observe that the viscous term has an additional 'curvature term' $\left(\mathbf{u}_{n+1}^{h}-2 \mathbf{u}_{n}^{h}+\right.$ $\left.\mathbf{u}_{n-1}^{h}\right)$ compared to standard NSE stability estimates. It is these terms which act to penalize curvature in the BDF2LEREG method. Moreover, the viscous term suggests that $\epsilon \leq O(\nu)$ is most appropriate, otherwise the curvature terms could dominate the viscous dissipation. Our numerical experiment show that $\epsilon=O(\nu)$ works very well.

Proof. Set in (2.1) $\mathbf{v}^{h}=\mathcal{J}_{n+\theta}^{\epsilon}\left(\mathbf{u}^{h}\right)$. Then we have

$$
\begin{aligned}
\frac{1}{\triangle t} \| & \left.\| \begin{array}{c}
\mathbf{u}_{n+1}^{h} \\
\mathbf{u}_{n}^{h}
\end{array}\right]\left\|_{G}^{2}-\frac{1}{\triangle t}\right\|\left[\begin{array}{c}
\mathbf{u}_{n}^{h} \\
\mathbf{u}_{n-1}^{h}
\end{array}\right]\left\|_{G}^{2}+\frac{1}{4 \triangle t}\right\| \mathbf{u}_{n+1}^{h}-2 \mathbf{u}_{n}^{h}+\mathbf{u}_{n-1}^{h}\left\|_{F}^{2}+\nu\right\| \mathcal{J}_{n+\theta}^{\epsilon}\left(\nabla \mathbf{u}^{h}\right) \|^{2} \\
& =\left(\mathbf{f}_{n+\theta}, \mathcal{J}_{n+\theta}^{\epsilon}\left(\mathbf{u}^{h}\right)\right) .
\end{aligned}
$$


Applying Cauchy-Schwarz inequality to the right hand side and then taking sum from $n=1$ to $n=N-1$, we have the following inequality

$$
\begin{aligned}
& \left\|\left[\begin{array}{c}
\mathbf{u}_{N}^{h} \\
\mathbf{u}_{N-1}^{h}
\end{array}\right]\right\|_{G}^{2}+\frac{1}{4} \sum_{n=1}^{N-1}\left\|\mathbf{u}_{n+1}^{h}-2 \mathbf{u}_{n}^{h}+\mathbf{u}_{n-1}^{h}\right\|_{F}^{2}+\frac{\triangle t \nu}{2} \sum_{n=1}^{N-1}\left\|\mathcal{J}_{n+\theta}^{\epsilon}\left(\nabla \mathbf{u}^{h}\right)\right\|^{2} \\
& \leq\left\|\left[\begin{array}{c}
\mathbf{u}_{1}^{h} \\
\mathbf{u}_{0}^{h}
\end{array}\right]\right\|_{G}^{2}+\frac{\Delta t}{2 \nu} \sum_{n=1}^{N-1}\left\|\mathbf{f}_{n+\theta}\right\|^{2} .
\end{aligned}
$$

By Lemma 3.1, we have the energy estimate as follows

$$
\begin{gathered}
\left\|\mathbf{u}_{N}^{h}\right\|^{2}+\frac{1}{2 \theta+1} \sum_{n=1}^{N-1}\left\|\mathbf{u}_{n+1}^{h}-2 \mathbf{u}_{n}^{h}+\mathbf{u}_{n-1}^{h}\right\|_{F}^{2}+\frac{2 \triangle t \nu}{2 \theta+1} \sum_{n=1}^{N-1}\left\|\mathcal{J}_{n+\theta}^{\epsilon}\left(\nabla \mathbf{u}^{h}\right)\right\|^{2} \\
\leq \frac{2 \theta-1}{2 \theta+1}\left\|\mathbf{u}_{N-1}^{h}\right\|^{2}+\frac{4}{2 \theta+1}\left\|\left[\begin{array}{l}
\mathbf{u}_{1}^{h} \\
\mathbf{u}_{0}^{h}
\end{array}\right]\right\|_{G}^{2}+\frac{2 \triangle t}{\nu(2 \theta+1)} \sum_{n=1}^{N-1}\left\|\mathbf{f}_{n+\theta}\right\|_{*}^{2} .
\end{gathered}
$$

By induction, we obtain the following energy estimate

$$
\begin{gathered}
\left\|\mathbf{u}_{N}^{h}\right\|^{2}+\frac{1}{2 \theta+1} \sum_{n=1}^{N-1}\left\|\mathbf{u}_{n+1}^{h}-2 \mathbf{u}_{n}^{h}+\mathbf{u}_{n-1}^{h}\right\|_{F}^{2}+\frac{2 \triangle t \nu}{2 \theta+1} \sum_{n=1}^{N-1}\left\|\mathcal{J}_{n+\theta}^{\epsilon}\left(\nabla \mathbf{u}^{h}\right)\right\|^{2} \\
\leq\left(\frac{2 \theta-1}{2 \theta+1}\right)^{N}\left\|\mathbf{u}_{0}^{h}\right\|^{2}+\frac{4 N}{2 \theta+1}\left\|\left[\begin{array}{c}
\mathbf{u}_{1}^{h} \\
\mathbf{u}_{0}^{h}
\end{array}\right]\right\|_{G}^{2}+\frac{2 N \triangle t}{\nu(2 \theta+1)} \sum_{n=1}^{N-1}\left\|\mathbf{f}_{n+\theta}\right\|_{*}^{2} .
\end{gathered}
$$

$\square$

4. Error Analysis. In this section we present a detailed error analysis of the proposed methods. We prove here that the entire family of stabilized methods are optimally accurate. The use of the $G$-norms, and the extrapolation and interpolations operators, are critical for a smooth analysis of the entire family of methods.

Let $t_{n}=n \Delta t, n=0,1,2, \ldots, N_{T}$, and $T:=N_{T} \Delta t$. Denote $\mathbf{u}_{n}=\mathbf{u}\left(t_{n}\right)$. We introduce the following discrete norms:

$$
\||\mathbf{v}|\|_{m, k}:=\left(\sum_{n=0}^{N_{T}}\left\|\mathbf{v}_{n}\right\|_{k}^{m} \Delta t\right)^{1 / m}, \quad\||\mathbf{v}|\|_{\infty, k}=\max _{0 \leq n \leq N_{T}}\left\|\mathbf{v}_{n}\right\|_{k} .
$$

In order to establish the optimal asymptotic error estimates for the approximation we assume that the true solution satisfies the following regularity

$$
\begin{gathered}
\mathbf{u} \in L^{\infty}\left(0, T ; H^{1}(\Omega)\right) \cap H^{1}\left(0, T ; H^{k+1}(\Omega)\right) \cap H^{3}\left(0, T ; L^{2}(\Omega)\right) \cap H^{2}\left(0, T ; H^{1}(\Omega)\right), \\
p \in L^{2}\left(0, T ; H^{s+1}(\Omega)\right) \cap H^{2}\left(0, T ; L^{2}(\Omega)\right), \text { and } \mathrm{f} \in L^{2}\left(0, T ; L^{2}(\Omega)\right) .
\end{gathered}
$$

We stress that this regularity is assumed, and regularity of Navier-Stokes solutions remains an open problem. Expected convergence rates will be reduced if less regularity exists for a particular problem. Deeper discussion of the regularity issues involved can be found in $[16,14]$.

THEOREM 4.1. Let $\mathbf{u}^{h}$ solve (1.4), and $\mathbf{u}$ be the true Navier-Stokes solution of the same problem data, with assumed regularity stated above, and denote $\mathbf{e}_{n}=\mathbf{u}_{n}-\mathbf{u}_{n}^{h}$. There exists a positive constant $C$ independent of the mesh width $h$ and timestep $\Delta t$ such that 


$$
\begin{aligned}
\left\|\mathbf{e}_{N}\right\|^{2} & +\frac{1}{2 \theta+1} \sum_{n=1}^{N-1}\left\|\mathbf{e}_{n+1}-2 \mathbf{e}_{n}+\mathbf{e}_{n-1}\right\|_{F}^{2}+\frac{2}{2 \theta+1} \Delta t \sum_{n=1}^{N-1} \nu\left\|\mathcal{J}_{n+\theta}^{\epsilon}(\nabla \mathbf{e})\right\|^{2} \\
\leq & \exp \left(C \nu^{-1} T\right)\left[\left(\frac{2 \theta-1}{2 \theta+1}\right)^{N}\left\|\mathbf{e}_{0}\right\|^{2}+C\left(1-\left(\frac{2 \theta-1}{2 \theta+1}\right)^{N}\right)\left(\left\|\mathbf{e}_{1}\right\|^{2}+\left\|\mathbf{e}_{0}\right\|^{2}\right)\right. \\
& +C\left(1-\left(\frac{2 \theta-1}{2 \theta+1}\right)^{N}\right)\left(\nu^{-1}(\Delta t)^{4}\left\|\left|p_{t t}\right|\right\|_{2,0}^{2}+\nu^{-1} h^{2 s+2}\||p|\|_{2, s+1}^{2}\right. \\
& +\nu^{-1}(\Delta t)^{4}\left\|\left|\mathbf{u}_{t t t}\right|\right\|_{2,0}^{2}+\nu(\Delta t)^{4}\left\|\left|\nabla \mathbf{u}_{t t}\right|\right\|_{2,0}^{2}+\nu^{-1}(\Delta t)^{4}\left\|\left|\nabla \mathbf{u}_{t t}\right|\right\|_{2,0}^{2} \\
& +\nu^{-1}(\Delta t)^{4}\left\|\left|\nabla \mathbf{u}\left\|_{\infty, 0}^{2}\right\|\right| \nabla \mathbf{u}_{t t}\left|\left\|_{2,0}^{2}+\nu^{-1}(\Delta t)^{4}\right\| \nabla \mathbf{u}\left\|_{\infty, 0}^{2}\right\|\right| \nabla \mathbf{u}_{t t} \mid\right\|_{2,0}^{2} \\
& \left.\left.+\nu^{-1} h^{2 k+2}\left\|\left|\mathbf{u}_{t}\right|\right\|_{2, k+1}^{2}+\nu h^{2 k}\||\mathbf{u}|\|_{2, k+1}^{2}+\nu^{-1} h^{2 k}\||\nabla \mathbf{u}|\|_{\infty, 0}^{2}\||\mathbf{u}|\|_{2, k+1}^{2}\right)\right] .
\end{aligned}
$$

Corollary 4.2. Consider Taylor-Hood approximation elements for the spatial discretization, i.e. $\left(X^{h}, Q^{h}\right)=\left(P_{2}, P_{1}\right)$. Then we have optimal accuracy in space and time:

$$
\begin{gathered}
\left\|\mathbf{e}_{N}\right\|^{2}+\frac{1}{2 \theta+1} \sum_{n=1}^{N-1}\left\|\mathbf{e}_{n+1}-2 \mathbf{e}_{n}+\mathbf{e}_{n-1}\right\|_{F}^{2}+\frac{2}{2 \theta+1} \Delta t \sum_{n=1}^{N-1} \nu\left\|\mathcal{J}_{n+\theta}^{\epsilon}(\nabla \mathbf{e})\right\|^{2} \\
\leq C\left((\Delta t)^{4}+h^{4}+\left\|\mathbf{e}_{0}\right\|^{2}+\left\|\mathbf{e}_{1}\right\|^{2}\right)
\end{gathered}
$$

REMARK 4.1. The first approximation of the velocity $\mathbf{u}_{0}^{h}$ needs to be weakly divergence free for the methods to be stable. For the methods to be optimal order convergent, it also needs to be interpolated in $V_{h}$ in a way such that $\left\|\mathbf{e}_{0}\right\|$ is $O\left(h^{2}\right)$ accurate or better.

For $\theta>\frac{1}{2}$, (1.4) are three-step methods. To achieve optimal order accuracy, a second order, two-step method, such as Crank-Nicolson method, is needed to compute the second approximation $u_{1}^{h}$, so that $\left\|e_{1}\right\|$ is $O\left((\Delta t)^{2}+h^{2}\right)$ accurate or better.

Proof. At time $t_{n+\theta}=(n+\theta) \triangle t$, the true solution of the NSE satisfies:

$$
\begin{aligned}
& \left(\frac{\left(\theta+\frac{1}{2}\right) \mathbf{u}_{n+1}-2 \theta \mathbf{u}_{n}+\left(\theta-\frac{1}{2}\right) \mathbf{u}_{n-1}}{\triangle t}, \mathbf{v}^{h}\right)+\nu\left(\mathcal{J}_{n+\theta}^{\epsilon}(\nabla \mathbf{u}), \nabla \mathbf{v}^{h}\right) \\
& \quad+b^{*}\left(\mathcal{H}_{n+\theta}(\mathbf{u}), \mathcal{J}_{n+\theta}^{\epsilon}(\mathbf{u}), \mathbf{v}^{h}\right)-\left(p_{n+\theta}, \nabla \cdot \mathbf{v}^{h}\right)=\left(f_{n+\theta}, \mathbf{v}^{h}\right)+\operatorname{Intp}\left(\mathbf{u}_{n+\theta} ; \mathbf{v}^{h}\right)
\end{aligned}
$$

where

$$
\begin{aligned}
& \operatorname{Intp}\left(\mathbf{u}_{n+\theta} ; \mathbf{v}^{h}\right)=\left(\frac{\left(\theta+\frac{1}{2}\right) \mathbf{u}_{n+1}-2 \theta \mathbf{u}_{n}+\left(\theta-\frac{1}{2}\right) \mathbf{u}_{n-1}}{\triangle t}-\mathbf{u}_{t}\left(t_{n+\theta}\right), \mathbf{v}^{h}\right) \\
& \quad+\nu\left(\nabla\left(\mathcal{J}_{n+\theta}^{\epsilon}(\mathbf{u})-\mathbf{u}_{n+\theta}\right), \nabla \mathbf{v}^{h}\right)+b^{*}\left(\mathcal{H}_{n+\theta}(\mathbf{u})-\mathbf{u}_{n+\theta}, \mathcal{J}_{n+\theta}^{\epsilon}(\mathbf{u}), \mathbf{v}^{h}\right) \\
& \quad+b^{*}\left(\mathbf{u}_{n+\theta}, \mathcal{J}_{n+\theta}^{\epsilon}(\mathbf{u})-\mathbf{u}_{n+\theta}, \mathbf{v}^{h}\right)
\end{aligned}
$$

Decompose the error into pieces in and out of the finite element space, by adding and subtracting an appropriate interpolant, and grouping terms.

$$
\mathbf{e}_{n}=\mathbf{u}_{n}-\mathbf{u}_{n}^{h}=\left(\mathbf{u}_{n}-I^{h} \mathbf{u}_{n}\right)+\left(I^{h} \mathbf{u}_{n}-\mathbf{u}_{n}^{h}\right)=\boldsymbol{\eta}_{n}+\boldsymbol{\xi}_{n}^{h},
$$


where $I^{h} \mathbf{u}_{n} \in V^{h}$ is an interpolant of $\mathbf{u}_{n}$ in $V^{h}$. Subtracting (2.1) from (4.2) gives

$$
\begin{aligned}
& \left(\frac{\left(\theta+\frac{1}{2}\right) \mathbf{e}_{n+1}-2 \theta \mathbf{e}_{n}+\left(\theta-\frac{1}{2}\right) \mathbf{e}_{n-1}}{\triangle t}, \mathbf{v}^{h}\right)+\nu\left(\mathcal{J}_{n+\theta}^{\epsilon}(\nabla \mathbf{e}), \nabla \mathbf{v}^{h}\right) \\
& +b^{*}\left(\mathcal{H}_{n+\theta}(\mathbf{u}), \mathcal{J}_{n+\theta}^{\epsilon}(\mathbf{e}), \mathbf{v}^{h}\right)=\operatorname{Intp}\left(\mathbf{u}_{n+\theta} ; \mathbf{v}^{h}\right)-\left(\mathcal{J}_{n+\theta}^{\epsilon}(p)-p_{n+\theta}, \nabla \cdot \mathbf{v}^{h}\right) \\
& +\left(\mathcal{J}_{n+\theta}^{\epsilon}(p)-q^{h}, \nabla \cdot \mathbf{v}^{h}\right)+b^{*}\left(\mathcal{H}_{n+\theta}(\mathbf{e}), \mathcal{J}_{n+\theta}^{\epsilon}\left(\mathbf{u}^{h}\right), \mathbf{v}^{h}\right),
\end{aligned}
$$

which, with the error splitting (4.4), can be rewritten as,

$$
\begin{aligned}
( & \left.\frac{\left(\theta+\frac{1}{2}\right) \boldsymbol{\xi}_{n+1}^{h}-2 \theta \boldsymbol{\xi}_{n}^{h}+\left(\theta-\frac{1}{2}\right) \boldsymbol{\xi}_{n-1}^{h}}{\triangle t}, \mathbf{v}^{h}\right)+\nu\left(\mathcal{J}_{n+\theta}^{\epsilon}\left(\nabla \boldsymbol{\xi}^{h}\right), \nabla \mathbf{v}^{h}\right) \\
& +b^{*}\left(\mathcal{H}_{n+\theta}(\mathbf{u}), \mathcal{J}_{n+\theta}^{\epsilon}\left(\boldsymbol{\xi}^{h}\right), \mathbf{v}^{h}\right) \\
= & \operatorname{Intp}\left(\mathbf{u}_{n+\theta} ; \mathbf{v}^{h}\right)-\left(\mathcal{J}_{n+\theta}^{\epsilon}(p)-p_{n+\theta}, \nabla \cdot \mathbf{v}^{h}\right)+\left(\mathcal{J}_{n+\theta}^{\epsilon}(p)-q^{h}, \nabla \cdot \mathbf{v}^{h}\right) \\
& -\left(\frac{\left(\theta+\frac{1}{2}\right) \boldsymbol{\eta}_{n+1}-2 \theta \boldsymbol{\eta}_{n}+\left(\theta-\frac{1}{2}\right) \boldsymbol{\eta}_{n-1}}{\triangle t}, \mathbf{v}^{h}\right)-\nu\left(\mathcal{J}_{n+\theta}^{\epsilon}(\nabla \boldsymbol{\eta}), \nabla \mathbf{v}^{h}\right) \\
& -b^{*}\left(\mathcal{H}_{n+\theta}(\mathbf{u}), \mathcal{J}_{n+\theta}^{\epsilon}(\boldsymbol{\eta}), \mathbf{v}^{h}\right)+b^{*}\left(\mathcal{H}_{n+\theta}\left(\boldsymbol{\xi}^{h}\right), \mathcal{J}_{n+\theta}^{\epsilon}\left(\mathbf{u}^{h}\right), \mathbf{v}^{h}\right) \\
& +b^{*}\left(\mathcal{H}_{n+\theta}(\boldsymbol{\eta}), \mathcal{J}_{n+\theta}^{\epsilon}\left(\mathbf{u}^{h}\right), \mathbf{v}^{h}\right) .
\end{aligned}
$$

Setting $\mathbf{v}^{h}=\mathcal{J}_{n+\theta}^{\epsilon}\left(\boldsymbol{\xi}^{h}\right)$ and using the skew symmetry of the trilinear term, (4.5) becomes

$$
\begin{aligned}
& \frac{1}{\triangle t}\left\|\left[\begin{array}{c}
\boldsymbol{\xi}_{n+1}^{h} \\
\boldsymbol{\xi}_{n}^{h}
\end{array}\right]\right\|_{G}^{2}-\frac{1}{\triangle t}\left\|\left[\begin{array}{c}
\boldsymbol{\xi}_{n}^{h} \\
\boldsymbol{\xi}_{n-1}^{h}
\end{array}\right]\right\|_{G}^{2}+\frac{1}{4 \triangle t}\left\|\boldsymbol{\xi}_{n+1}^{h}-2 \boldsymbol{\xi}_{n}^{h}+\boldsymbol{\xi}_{n-1}^{h}\right\|_{F}^{2}+\nu\left\|\mathcal{J}_{n+\theta}^{\epsilon}\left(\nabla \boldsymbol{\xi}^{h}\right)\right\|^{2} \quad \text { (4.6) } \\
& =\operatorname{Intp}\left(\mathbf{u}_{n+\theta} ; \mathcal{J}_{n+\theta}^{\epsilon}\left(\boldsymbol{\xi}^{h}\right)\right)-\left(\mathcal{J}_{n+\theta}^{\epsilon}(p)-p_{n+\theta}, \nabla \cdot \mathcal{J}_{n+\theta}^{\epsilon}\left(\boldsymbol{\xi}^{h}\right)\right) \\
& \quad+\left(\mathcal{J}_{n+\theta}^{\epsilon}(p)-q^{h}, \nabla \cdot\left(\mathcal{J}_{n+\theta}^{\epsilon}\left(\boldsymbol{\xi}^{h}\right)\right)-\left(\frac{\left(\theta+\frac{1}{2}\right) \boldsymbol{\eta}_{n+1}-2 \theta \boldsymbol{\eta}_{n}+\left(\theta-\frac{1}{2}\right) \boldsymbol{\eta}_{n-1}}{\triangle t}, \mathcal{J}_{n+\theta}^{\epsilon}\left(\boldsymbol{\xi}^{h}\right)\right)\right. \\
& \quad-\nu\left(\mathcal{J}_{n+\theta}^{\epsilon}(\nabla \boldsymbol{\eta}), \mathcal{J}_{n+\theta}^{\epsilon}\left(\nabla \boldsymbol{\xi}^{h}\right)\right)-b^{*}\left(\mathcal{H}_{n+\theta}(\mathbf{u}), \mathcal{J}_{n+\theta}^{\epsilon}(\boldsymbol{\eta}), \mathcal{J}_{n+\theta}^{\epsilon}\left(\boldsymbol{\xi}^{h}\right)\right) \\
& \quad+b^{*}\left(\mathcal{H}_{n+\theta}\left(\boldsymbol{\xi}^{h}\right), \mathcal{J}_{n+\theta}^{\epsilon}\left(\mathbf{u}^{h}\right), \mathcal{J}_{n+\theta}^{\epsilon}\left(\boldsymbol{\xi}^{h}\right)\right)+b^{*}\left(\mathcal{H}_{n+\theta}(\boldsymbol{\eta}), \mathcal{J}_{n+\theta}^{\epsilon}\left(\mathbf{u}^{h}\right), \mathcal{J}_{n+\theta}^{\epsilon}\left(\boldsymbol{\xi}^{h}\right)\right) .
\end{aligned}
$$

Using Cauchy-Schwarz and Young's inequalities, followed by Taylor's theorem, we obtain the following bounds on the pressure terms:

$$
\begin{aligned}
&\left(\mathcal{J}_{n+\theta}^{\epsilon}(p)-p_{n+\theta}, \nabla \cdot \mathcal{J}_{n+\theta}^{\epsilon}\left(\boldsymbol{\xi}^{h}\right)\right) \\
& \leq \frac{\nu}{64}\left\|\mathcal{J}_{n+\theta}^{\epsilon}\left(\nabla \boldsymbol{\xi}^{h}\right)\right\|^{2}+C \nu^{-1}\left\|\mathcal{J}_{n+\theta}^{\epsilon}(p)-p_{n+\theta}\right\|^{2} \\
& \leq \frac{\nu}{64}\left\|\mathcal{J}_{n+\theta}^{\epsilon}\left(\nabla \boldsymbol{\xi}^{h}\right)\right\|^{2}+C \nu^{-1}\left\|\theta p_{n+1}+(1-\theta) p_{n}-p_{n+\theta}\right\|^{2}+C \nu^{-3} \epsilon^{2} \theta^{2}\left\|p_{n+1}-2 p_{n}+p_{n-1}\right\|^{2} \\
& \leq \frac{\nu}{64}\left\|\nabla \mathcal{J}_{n+\theta}^{\epsilon}\left(\boldsymbol{\xi}^{h}\right)\right\|^{2}+C \nu^{-1} \theta^{2}(1-\theta)^{2} \Delta t^{3} \int_{t_{n}}^{t_{n+1}}\left\|p_{t t}\right\|^{2} d t+C \nu^{-3} \epsilon^{2} \theta^{2} \Delta t^{3} \int_{t_{n-1}}^{t_{n+1}}\left\|p_{t t}\right\|^{2} d t \\
&\left(\mathcal{J}_{n+\theta}^{\epsilon}(p)-q^{h}, \nabla \cdot \mathcal{J}_{n+\theta}^{\epsilon}\left(\boldsymbol{\xi}^{h}\right)\right) \leq \frac{\nu}{64}\left\|\mathcal{J}_{n+\theta}^{\epsilon}\left(\nabla \boldsymbol{\xi}^{h}\right)\right\|^{2}+C \nu^{-1}\left\|\mathcal{J}_{n+\theta}^{\epsilon}(p)-q^{h}\right\|^{2} \\
& \leq \frac{\nu}{64}\left\|\mathcal{J}_{n+\theta}^{\epsilon}\left(\nabla \boldsymbol{\xi}^{h}\right)\right\|^{2}+C \nu^{-1}\left(\left\|\mathcal{J}_{n+\theta}^{\epsilon}(p)-p_{n+\theta}\right\|^{2}+\left\|p_{n+\theta}-q^{h}\right\|^{2}\right) \\
& \leq \frac{\nu}{64}\left\|\mathcal{J}_{n+\theta}^{\epsilon}\left(\nabla \boldsymbol{\xi}^{h}\right)\right\|^{2}+C \nu^{-1} \theta^{2}(1-\theta)^{2} \Delta t^{3} \int_{t_{n}}^{t_{n+1}}\left\|p_{t t}\right\|^{2} d t \\
& \quad+C \nu^{-3} \epsilon^{2} \theta^{2} \Delta t^{3} \int_{t_{n-1}}^{t_{n+1}}\left\|p_{t t}\right\|^{2} d t+C \nu^{-1} h^{2 s+2}\left\|p_{n+\theta}\right\|_{s+1}^{2} .
\end{aligned}
$$


Next, we bound Intp $\left(\mathbf{u}_{n+\theta} ; \mathcal{J}_{n+\theta}^{\epsilon}\left(\boldsymbol{\xi}^{h}\right)\right)$. Cauchy-Schwarz, Young, and Poincare inequalities, along with Taylor's theorem provide

$$
\begin{aligned}
& \left(\frac{\left(\theta+\frac{1}{2}\right) \mathbf{u}_{n+1}-2 \theta \mathbf{u}_{n}+\left(\theta-\frac{1}{2}\right) \mathbf{u}_{n-1}}{\triangle t}-\mathbf{u}_{t}\left(t_{n+\theta}\right), \mathcal{J}_{n+\theta}^{\epsilon}\left(\boldsymbol{\xi}^{h}\right)\right) \\
& \leq \frac{\nu}{64}\left\|\mathcal{J}_{n+\theta}^{\epsilon}\left(\nabla \boldsymbol{\xi}^{h}\right)\right\|^{2}+C \nu^{-1}\left\|\frac{\left(\theta+\frac{1}{2}\right) \mathbf{u}_{n+1}-2 \theta \mathbf{u}_{n}+\left(\theta-\frac{1}{2}\right) \mathbf{u}_{n-1}}{\triangle t}-\mathbf{u}_{t}\left(t_{n+\theta}\right)\right\|^{2} \\
& \leq \frac{\nu}{64}\left\|\mathcal{J}_{n+\theta}^{\epsilon}\left(\nabla \boldsymbol{\xi}^{h}\right)\right\|^{2}+C \nu^{-1} \theta^{6}(\Delta t)^{3} \int_{t_{n-1}}^{t_{n+1}}\left\|\mathbf{u}_{t t t}\right\|^{2} d t
\end{aligned}
$$

For the viscous term in Intp, we apply Cauchy-Schwarz and Young's inequalities, and Taylor's theorem to obtain the bound

$$
\begin{aligned}
\nu\left(\nabla\left(\mathcal{J}_{n+\theta}^{\epsilon}(\mathbf{u})-\mathbf{u}_{n+\theta}\right), \nabla \mathcal{J}_{n+\theta}^{\epsilon}\left(\boldsymbol{\xi}^{h}\right)\right) \\
\leq \frac{\nu}{64}\left\|\mathcal{J}_{n+\theta}^{\epsilon}\left(\nabla \boldsymbol{\xi}^{h}\right)\right\|^{2}+C \nu\left\|\nabla\left(\mathcal{J}_{n+\theta}^{\epsilon}(\mathbf{u})-\mathbf{u}_{n+\theta}\right)\right\|^{2} \\
\leq \frac{\nu}{64}\left\|\mathcal{J}_{n+\theta}^{\epsilon}\left(\nabla \boldsymbol{\xi}^{h}\right)\right\|^{2}+C \nu\left\|\nabla\left(\theta \mathbf{u}_{n+1}+(1-\theta) \mathbf{u}_{n}-\mathbf{u}_{n+\theta}\right)\right\|^{2} \\
\quad+C \nu^{-1} \epsilon^{2} \theta^{2}\left\|\nabla\left(\mathbf{u}_{n+1}-2 \mathbf{u}_{n}+\mathbf{u}_{n-1}\right)\right\|^{2} \\
\leq \frac{\nu}{64}\left\|\nabla \mathcal{J}_{n+\theta}^{\epsilon}\left(\boldsymbol{\xi}^{h}\right)\right\|^{2}+C \nu \theta^{2}(1-\theta)^{2}(\Delta t)^{3} \int_{t_{n}}^{t_{n+1}}\left\|\nabla \mathbf{u}_{t t}\right\|^{2} d t \\
\quad+C \nu^{-1} \epsilon^{2} \theta^{2}(\Delta t)^{3} \int_{t_{n-1}}^{t_{n+1}}\left\|\nabla \mathbf{u}_{t t}\right\|^{2} d t .
\end{aligned}
$$

For the first trilinear term, we first use standard Sobolev bounds together with Hölder's inequality, followed by Young's inequality and Taylor's theorem, which yields

$$
\begin{aligned}
& b^{*}\left(\mathcal{H}_{n+\theta}(\mathbf{u})-\mathbf{u}_{n+\theta}, \mathcal{J}_{n+\theta}^{\epsilon}(\mathbf{u}), \mathcal{J}_{n+\theta}^{\epsilon}\left(\boldsymbol{\xi}^{h}\right)\right) \\
& \quad \leq C\left\|\nabla\left(\mathcal{H}_{n+\theta}(\mathbf{u})-\mathbf{u}_{n+\theta}\right)\right\|\left\|\mathcal{J}_{n+\theta}^{\epsilon}(\nabla \mathbf{u})\right\|\left\|\mathcal{J}_{n+\theta}^{\epsilon}\left(\nabla \boldsymbol{\xi}^{h}\right)\right\| \\
& \quad \leq \frac{\nu}{64}\left\|\mathcal{J}_{n+\theta}^{\epsilon}\left(\nabla \boldsymbol{\xi}^{h}\right)\right\|^{2}+C \nu^{-1}\left\|\nabla\left(\mathcal{H}_{n+\theta}(\mathbf{u})-\mathbf{u}_{n+\theta}\right)\right\|^{2}\left\|\mathcal{J}_{n+\theta}^{\epsilon}(\nabla \mathbf{u})\right\|^{2} \\
& \quad \leq \frac{\nu}{64}\left\|\mathcal{J}_{n+\theta}^{\epsilon}\left(\nabla \boldsymbol{\xi}^{h}\right)\right\|^{2}+C \nu^{-1} \theta^{2}(1+\theta)^{2}(\Delta t)^{3}\left\|\mathcal{J}_{n+\theta}^{\epsilon}(\nabla \mathbf{u})\right\|^{2} \int_{t_{n-1}}^{t_{n+1}}\left\|\nabla \mathbf{u}_{t t}\right\|^{2} d t
\end{aligned}
$$

Similarly,

$$
\begin{aligned}
& b^{*}\left(\mathbf{u}_{n+\theta}, \mathcal{J}_{n+\theta}^{\epsilon}(\mathbf{u})-\mathbf{u}_{n+\theta}, \mathcal{J}_{n+\theta}^{\epsilon}\left(\boldsymbol{\xi}^{h}\right)\right) \\
& \leq C\left\|\nabla \mathbf{u}_{n+\theta}\right\|\left\|\nabla\left(\mathcal{J}_{n+\theta}^{\epsilon}(\mathbf{u})-\mathbf{u}_{n+\theta}\right)\right\|\left\|\mathcal{J}_{n+\theta}^{\epsilon}\left(\nabla \boldsymbol{\xi}^{h}\right)\right\| \\
& \leq \frac{\nu}{64}\left\|\mathcal{J}_{n+\theta}^{\epsilon}\left(\nabla \boldsymbol{\xi}^{h}\right)\right\|^{2}+C \nu^{-1}\left\|\nabla \mathbf{u}_{n+\theta}\right\|^{2}\left\|\nabla\left(\mathcal{J}_{n+\theta}^{\epsilon}(\mathbf{u})-\mathbf{u}_{n+\theta}\right)\right\|^{2} \\
& \leq \frac{\nu}{64}\left\|\mathcal{J}_{n+\theta}^{\epsilon}\left(\nabla \boldsymbol{\xi}^{h}\right)\right\|^{2}+C \nu^{-1} \theta^{2}(1-\theta)^{2}(\Delta t)^{3}\left\|\nabla \mathbf{u}_{n+\theta}\right\|^{2} \int_{t_{n}}^{t_{n+1}}\left\|\nabla \mathbf{u}_{t t}\right\|^{2} d t \\
& \quad+C \nu^{-3} \epsilon^{2} \theta^{2}(\Delta t)^{3}\left\|\nabla \mathbf{u}_{n+\theta}\right\|^{2} \int_{t_{n-1}}^{t_{n+1}}\left\|\nabla \mathbf{u}_{t t}\right\|^{2} d t .
\end{aligned}
$$

We then bound the other terms on the right hand side of (4.6) using Cauchy-Schwarz and fundamental theorem of Calculus,

$$
\left(\frac{\left(\theta+\frac{1}{2}\right) \boldsymbol{\eta}_{n+1}-2 \theta \boldsymbol{\eta}_{n}+\left(\theta-\frac{1}{2}\right) \boldsymbol{\eta}_{n-1}}{\triangle t}, \mathcal{J}_{n+\theta}^{\epsilon}\left(\boldsymbol{\xi}^{h}\right)\right)
$$




$$
\begin{aligned}
& \leq\left\|\frac{\left(\theta+\frac{1}{2}\right) \boldsymbol{\eta}_{n+1}-2 \theta \boldsymbol{\eta}_{n}+\left(\theta-\frac{1}{2}\right) \boldsymbol{\eta}_{n-1}}{\triangle t}\right\|\left\|\mathcal{J}_{n+\theta}^{\epsilon}\left(\boldsymbol{\xi}^{h}\right)\right\| \\
& \leq C\left\|\frac{\frac{1}{2}\left(\boldsymbol{\eta}_{n+1}-\boldsymbol{\eta}_{n-1}\right)+\theta\left(\boldsymbol{\eta}_{n+1}-\boldsymbol{\eta}_{n}\right)-\theta\left(\boldsymbol{\eta}_{n}-\boldsymbol{\eta}_{n-1}\right)}{\triangle t}\right\|\left\|\mathcal{J}_{n+\theta}^{\epsilon}\left(\nabla \boldsymbol{\xi}^{h}\right)\right\| \\
& \leq C\left\|\frac{1}{\triangle t}\left(\frac{1}{2} \int_{t_{n-1}}^{t_{n+1}} \boldsymbol{\eta}_{t} d t+\theta \int_{t_{n}}^{t_{n+1}} \boldsymbol{\eta}_{t} d t-\theta \int_{t_{n-1}}^{t^{n}} \boldsymbol{\eta}_{t} d t\right)\right\|\left\|\mathcal{J}_{n+\theta}^{\epsilon}\left(\nabla \boldsymbol{\xi}^{h}\right)\right\| \\
& \leq C\left(\left\|\frac{1}{2 \triangle t} \int_{t_{n-1}}^{t_{n+1}}\left|\boldsymbol{\eta}_{t}\right| d t\right\|+\left\|\frac{1}{\triangle t} \theta \int_{t_{n}}^{t_{n+1}}\left|\boldsymbol{\eta}_{t}\right| d t\right\|+\left\|\frac{1}{\triangle t} \theta \int_{t_{n-1}}^{t^{n}}\left|\boldsymbol{\eta}_{t}\right| d t\right\|\right)\left\|\mathcal{J}_{n+\theta}^{\epsilon}\left(\nabla \boldsymbol{\xi}^{h}\right)\right\| \\
& \leq C \nu^{-1}\left(\theta^{2}+4\right)\left\|\frac{1}{\triangle t} \int_{t_{n-1}}^{t_{n+1}}\left|\boldsymbol{\eta}_{t}\right| d t\right\|^{2}+\frac{\nu}{64}\left\|\mathcal{J}_{n+\theta}^{\epsilon}\left(\nabla \boldsymbol{\xi}^{h}\right)\right\|^{2} \\
& \leq \frac{C\left(\theta^{2}+4\right)}{\nu \triangle t} \int_{t_{n-1}}^{t_{n+1}}\left\|\boldsymbol{\eta}_{t}\right\|^{2} d t+\frac{\nu}{64}\left\|\mathcal{J}_{n+\theta}^{\epsilon}\left(\nabla \boldsymbol{\xi}^{h}\right)\right\|^{2} .
\end{aligned}
$$

For the remaining viscous term, we apply Cauchy-Schwarz and Young's inequalities, and expand the $\mathcal{J}_{n+\theta}^{\epsilon}$ operator to obtain

$$
\begin{aligned}
& \nu\left(\mathcal{J}_{n+\theta}^{\epsilon}(\nabla \boldsymbol{\eta}), \mathcal{J}_{n+\theta}^{\epsilon}\left(\nabla \boldsymbol{\xi}^{h}\right)\right) \leq \nu\left\|\mathcal{J}_{n+\theta}^{\epsilon}(\nabla \boldsymbol{\eta})\right\|\left\|\mathcal{J}_{n+\theta}^{\epsilon}\left(\nabla \boldsymbol{\xi}^{h}\right)\right\| \\
& \leq C \nu\left(\left(\theta+\frac{\epsilon \theta}{\nu}\right)^{2}\left\|\nabla \boldsymbol{\eta}_{n+1}\right\|^{2}+\left(1-\theta-\frac{2 \epsilon \theta}{\nu}\right)^{2}\left\|\nabla \boldsymbol{\eta}_{n}\right\|^{2}+\frac{\epsilon^{2} \theta^{2}}{\nu^{2}}\left\|\nabla \boldsymbol{\eta}_{n-1}\right\|^{2}\right) \\
& \quad+\frac{\nu}{64}\left\|\mathcal{J}_{n+\theta}^{\epsilon}\left(\nabla \boldsymbol{\xi}^{h}\right)\right\|^{2} .
\end{aligned}
$$

We estimate the remaining nonlinear terms in the usual way (see, e.g. [16, 10, 25]), and then expand the operators and use the triangle inequality, yielding

$$
\begin{aligned}
b^{*} & \left(\mathcal{H}_{n+\theta}(\mathbf{u}), \mathcal{J}_{n+\theta}^{\epsilon}(\boldsymbol{\eta}), \mathcal{J}_{n+\theta}^{\epsilon}\left(\boldsymbol{\xi}^{h}\right)\right) \\
\leq & C\left\|\mathcal{H}_{n+\theta}(\nabla \mathbf{u})\right\|\left\|\mathcal{J}_{n+\theta}^{\epsilon}(\nabla \boldsymbol{\eta})\right\|\left\|\mathcal{J}_{n+\theta}^{\epsilon}\left(\nabla \boldsymbol{\xi}^{h}\right)\right\| \\
\leq & C \nu^{-1}\left((\theta+1)^{2}\left\|\nabla \mathbf{u}_{n}\right\|^{2}+\theta^{2}\left\|\nabla \mathbf{u}_{n-1}\right\|^{2}\right)\left(\left(\theta+\frac{\epsilon \theta}{\nu}\right)^{2}\left\|\nabla \boldsymbol{\eta}_{n+1}\right\|^{2}\right. \\
& \left.+\left(1-\theta-\frac{2 \epsilon \theta}{\nu}\right)^{2}\left\|\nabla \boldsymbol{\eta}_{n}\right\|^{2}+\frac{\epsilon^{2} \theta^{2}}{\nu^{2}}\left\|\nabla \boldsymbol{\eta}_{n-1}\right\|^{2}\right)+\frac{\nu}{64}\left\|\mathcal{J}_{n+\theta}^{\epsilon}\left(\nabla \boldsymbol{\xi}^{h}\right)\right\|^{2},
\end{aligned}
$$

and

$$
\begin{aligned}
& b^{*}\left(\mathcal{H}_{n+\theta}(\boldsymbol{\eta}), \mathcal{J}_{n+\theta}^{\epsilon}\left(\mathbf{u}^{h}\right), \mathcal{J}_{n+\theta}^{\epsilon}\left(\boldsymbol{\xi}^{h}\right)\right) \\
& \leq C\left\|\mathcal{H}_{n+\theta}(\nabla \boldsymbol{\eta})\right\|\left\|\mathcal{J}_{n+\theta}^{\epsilon}\left(\nabla \mathbf{u}^{h}\right)\right\|\left\|\mathcal{J}_{n+\theta}^{\epsilon}\left(\nabla \boldsymbol{\xi}^{h}\right)\right\| \\
& \leq C \nu^{-1}\left\|\mathcal{J}_{n+\theta}^{\epsilon}\left(\nabla \mathbf{u}^{h}\right)\right\|^{2}\left\|\mathcal{H}_{n+\theta}(\nabla \boldsymbol{\eta})\right\|^{2}+\frac{\nu}{64}\left\|\mathcal{J}_{n+\theta}^{\epsilon}\left(\nabla \boldsymbol{\xi}^{h}\right)\right\|^{2} \\
& \leq C \nu^{-1}\left(\left(\theta+\frac{\epsilon \theta}{\nu}\right)^{2}\left\|\nabla \mathbf{u}_{n+1}^{h}\right\|^{2}+\left(1-\theta-\frac{2 \epsilon \theta}{\nu}\right)^{2}\left\|\nabla \mathbf{u}_{n}^{h}\right\|^{2}+\frac{\epsilon^{2} \theta^{2}}{\nu^{2}}\left\|\nabla \mathbf{u}_{n-1}^{h}\right\|^{2}\right) \times \\
&\left((\theta+1)^{2}\left\|\nabla \boldsymbol{\eta}_{n}\right\|^{2}+\theta^{2}\left\|\nabla \boldsymbol{\eta}_{n-1}\right\|^{2}\right)+\frac{\nu}{64}\left\|\mathcal{J}_{n+\theta}^{\epsilon}\left(\nabla \boldsymbol{\xi}^{h}\right)\right\|^{2} .
\end{aligned}
$$

The last trilinear term is bounded using some additional regularity of the true solution, as

$$
\begin{aligned}
& b^{*}\left(\mathcal{H}_{n+\theta}\left(\boldsymbol{\xi}^{h}\right), \mathcal{J}_{n+\theta}^{\epsilon}\left(\mathbf{u}^{h}\right), \mathcal{J}_{n+\theta}^{\epsilon}\left(\boldsymbol{\xi}^{h}\right)\right) \\
& \quad \leq C\left\|\mathcal{J}_{n+\theta}^{\epsilon}\left(\nabla \mathbf{u}^{h}\right)\right\|_{\infty}\left\|\mathcal{H}_{n+\theta}\left(\boldsymbol{\xi}^{h}\right)\right\|\left\|\mathcal{J}_{n+\theta}^{\epsilon}\left(\nabla \boldsymbol{\xi}^{h}\right)\right\|
\end{aligned}
$$




$$
\begin{aligned}
+ & C\left\|\mathcal{J}_{n+\theta}^{\epsilon}\left(\mathbf{u}^{h}\right)\right\|_{\infty}\left\|\mathcal{H}_{n+\theta}\left(\boldsymbol{\xi}^{h}\right)\right\|\left\|\mathcal{J}_{n+\theta}^{\epsilon}\left(\nabla \boldsymbol{\xi}^{h}\right)\right\| \\
\leq C \nu^{-1}\left(\left\|\mathcal{J}_{n+\theta}^{\epsilon}\left(\nabla \mathbf{u}^{h}\right)\right\|_{\infty}^{2}+\left\|\mathcal{J}_{n+\theta}^{\epsilon}\left(\mathbf{u}^{h}\right)\right\|_{\infty}^{2}\right)\left((\theta+1)^{2}\left\|\boldsymbol{\xi}_{n}^{h}\right\|^{2}+\theta^{2}\left\|\boldsymbol{\xi}_{n-1}^{h}\right\|^{2}\right) & \\
& +\frac{\nu}{64}\left\|\mathcal{J}_{n+\theta}^{\epsilon}\left(\nabla \boldsymbol{\xi}^{h}\right)\right\|^{2} .
\end{aligned}
$$

After bounding all the terms on the right hand side of (4.6), we now take the sum from $n=1$ to $n=N-1$

$$
\begin{aligned}
&\left\|\left[\begin{array}{c}
\boldsymbol{\xi}_{N}^{h} \\
\boldsymbol{\xi}_{N-1}^{h}
\end{array}\right]\right\|_{G}^{2}+\frac{1}{4} \sum_{n=1}^{N-1}\left\|\boldsymbol{\xi}_{n+1}^{h}-2 \boldsymbol{\xi}_{n}^{h}+\boldsymbol{\xi}_{n-1}^{h}\right\|_{F}^{2}+\Delta t \sum_{n=1}^{N-1} \frac{\nu}{2}\left\|\mathcal{J}_{n+\theta}^{\epsilon}\left(\nabla \boldsymbol{\xi}^{h}\right)\right\|^{2} \\
& \leq\left\|\left[\begin{array}{c}
\boldsymbol{\xi}_{1}^{h} \\
\boldsymbol{\xi}_{0}^{h}
\end{array}\right]\right\|_{G}^{2}+C \Delta t \sum_{n=1}^{N-1}\left[\nu^{-1} \theta^{2}(1-\theta)^{2} \Delta t^{3} \int_{t_{n}}^{t_{n+1}}\left\|p_{t t}\right\|^{2} d t\right. \\
&+\nu^{-3} \epsilon^{2} \theta^{2}(\Delta t)^{3} \int_{t_{n-1}}^{t_{n+1}}\left\|p_{t t}\right\|^{2} d t+\nu^{-1} h^{2 s+2}\left\|p_{n+\theta}\right\|_{s+1}^{2} \\
&+\nu^{-1} \theta^{6}(\Delta t)^{3} \int_{t_{n-1}}^{t_{n+1}}\left\|\mathbf{u}_{t t t}\right\|^{2} d t+C \nu \theta^{2}(1-\theta)^{2}(\Delta t)^{3} \int_{t_{n}}^{t_{n+1}}\left\|\nabla \mathbf{u}_{t t}\right\|^{2} d t \\
&+C \nu^{-1} \epsilon^{2} \theta^{2}(\Delta t)^{3} \int_{t_{n-1}}^{t_{n+1}}\left\|\nabla \mathbf{u}_{t t}\right\|^{2} d t \\
&+C \nu^{-1} \theta^{2}(1+\theta)^{2}(\Delta t)^{3}\left\|\mathcal{J}_{n+\theta}^{\epsilon}(\nabla \mathbf{u})\right\|^{2} \int_{t_{n-1}}^{t_{n+1}}\left\|\nabla \mathbf{u}_{t t}\right\|^{2} d t \\
&+C \nu^{-1} \theta^{2}(1-\theta)^{2}(\Delta t)^{3}\left\|\nabla \mathbf{u}_{n+\theta}\right\|^{2} \int_{t_{n}}^{t_{n+1}}\left\|\nabla \mathbf{u}_{t t}\right\|^{2} d t \\
&+C \nu^{-3} \epsilon^{2} \theta^{2}(\Delta t)^{3}\left\|\nabla \mathbf{u}_{n+\theta}\right\|^{2} \int_{t_{n-1}}^{t_{n+1}}\left\|\nabla \mathbf{u}_{t t}\right\|^{2} d t+\frac{\left(\theta^{2}+4\right)}{\nu \triangle t} \int_{t_{n-1}}^{t_{n+1}}\left\|\boldsymbol{\eta}_{t}\right\|^{2} d t \\
&+\nu\left(\left(\theta+\frac{\epsilon \theta}{\nu}\right)^{2}\left\|\nabla \boldsymbol{\eta}_{n+1}\right\|^{2}+\left(1-\theta-\frac{2 \epsilon \theta}{\nu}\right)^{2}\left\|\nabla \boldsymbol{\eta}_{n}\right\|^{2}+\frac{\epsilon^{2} \theta^{2}}{\nu^{2}}\left\|\nabla \boldsymbol{\eta}_{n-1}\right\|^{2}\right) \\
&+\nu^{-1}\left((\theta+1)^{2}\left\|\nabla \mathbf{u}_{n}\right\|^{2}+\theta^{2}\left\|\nabla \mathbf{u}_{n-1}\right\|^{2}\right)\left(\left(\theta+\frac{\epsilon \theta}{\nu}\right)^{2}\left\|\nabla \boldsymbol{\eta}_{n+1}\right\|^{2}\right. \\
&\left.+\left(1-\theta-\frac{2 \epsilon \theta}{\nu}\right)^{2}\left\|\nabla \boldsymbol{\eta}_{n}\right\|^{2}+\frac{\epsilon^{2} \theta^{2}}{\nu^{2}}\left\|\nabla \boldsymbol{\eta}_{n-1}\right\|^{2}\right)+\nu^{-1}\left(\left(\theta+\frac{\epsilon \theta}{\nu}\right)^{2}\left\|\nabla \mathbf{u}_{n+1}^{h}\right\|^{2}\right. \\
&\left.+\left(1-\theta-\frac{2 \epsilon \theta}{\nu}\right)^{2}\left\|\nabla \mathbf{u}_{n}^{h}\right\|^{2}+\frac{\epsilon^{2} \theta^{2}}{\nu^{2}}\left\|\nabla \mathbf{u}_{n-1}^{h}\right\|^{2}\right) \times\left((\theta+1)^{2}\left\|\nabla \boldsymbol{\eta}_{n}\right\|^{2}+\theta^{2}\left\|\nabla \boldsymbol{\eta}_{n-1}\right\|^{2}\right) \\
&\left.+\nu^{-1}\left(\left\|\mathcal{J}_{n+\theta}^{\epsilon}\left(\nabla \mathbf{u}^{h}\right)\right\|_{\infty}^{2}+\left\|\mathcal{J}_{n+\theta}^{\epsilon}\left(\mathbf{u}^{h}\right)\right\|_{\infty}^{2}\right)\left((\theta+1)^{2}\left\|\boldsymbol{\xi}_{n}^{h}\right\|^{2}+\theta^{2}\left\|\boldsymbol{\xi}_{n-1}^{h}\right\|^{2}\right)\right]
\end{aligned}
$$

Using Lemma 3.1 and absorbing constants into $C$ we obtain

$$
\begin{aligned}
\left\|\boldsymbol{\xi}_{N}^{h}\right\|^{2} & +\frac{1}{2 \theta+1} \sum_{n=1}^{N-1}\left\|\boldsymbol{\xi}_{n+1}^{h}-2 \boldsymbol{\xi}_{n}^{h}+\boldsymbol{\xi}_{n-1}^{h}\right\|_{F}^{2}+\frac{2}{2 \theta+1} \Delta t \sum_{n=1}^{N-1} \nu\left\|\mathcal{J}_{n+\theta}^{\epsilon}\left(\nabla \boldsymbol{\xi}^{h}\right)\right\|^{2} \\
\leq & \left(\frac{2 \theta-1}{2 \theta+1}\right)^{N}\left\|\boldsymbol{\xi}_{0}^{h}\right\|^{2}+2\left(1-\left(\frac{2 \theta-1}{2 \theta+1}\right)^{N}\right)\left[\left\|\left[\begin{array}{l}
\boldsymbol{\xi}_{1}^{h} \\
\boldsymbol{\xi}_{0}^{h}
\end{array}\right]\right\|_{G}^{2}\right. \\
& +C\left(\nu^{-1}(\Delta t)^{4}\left\|\left|p_{t t}\right|\right\|_{2,0}^{2}+\nu^{-1} h^{2 s+2}\|||\|_{2, s+1}^{2}+\nu^{-1}(\Delta t)^{4}\left\|\mathbf{u}_{t t t} \mid\right\|_{2,0}^{2}\right. \\
& +\nu(\Delta t)^{4}\left\|\left|\nabla \mathbf{u}_{t t}\right|\right\|_{2,0}^{2}+\nu^{-1}(\Delta t)^{4}\left\|\left|\nabla \mathbf{u}_{t t}\right|\right\|_{2,0}^{2}+\nu^{-1}(\Delta t)^{4}\||\nabla \mathbf{u}|\|_{\infty, 0}^{2}\left\|\left|\nabla \mathbf{u}_{t t}\right|\right\|_{2,0}^{2}
\end{aligned}
$$




$$
\begin{aligned}
& +\nu^{-1}(\Delta t)^{4}\|\nabla \mathbf{u}\|_{\infty, 0}^{2}\left\|\left|\nabla \mathbf{u}_{t t}\right|\right\|_{2,0}^{2}+\nu^{-1} h^{2 k+2}\left\|\left|\mathbf{u}_{t}\right|\right\|_{2, k+1}^{2}+\nu h^{2 k}\||\mathbf{u}|\|_{2, k+1}^{2} \\
& \left.\left.+\nu^{-1} h^{2 k}\||\nabla \mathbf{u}|\|_{\infty, 0}^{2}\|\mathbf{u} \mid\|_{2, k+1}^{2}\right)+C \nu^{-1} \triangle t \sum_{n=0}^{N-1}\left\|\boldsymbol{\xi}_{n}^{h}\right\|^{2}\right] .
\end{aligned}
$$

Notice that

$$
0 \leq\left(\frac{2 \theta-1}{2 \theta+1}\right)^{N}<1, \text { for any } N \geq 0 .
$$

Then by Lemma 2.4 in $[9$, p. 176], we obtain

$$
\begin{aligned}
\left\|\boldsymbol{\xi}_{N}^{h}\right\|^{2} & +\frac{1}{2 \theta+1} \sum_{n=1}^{N-1}\left\|\boldsymbol{\xi}_{n+1}^{h}-2 \boldsymbol{\xi}_{n}^{h}+\boldsymbol{\xi}_{n-1}^{h}\right\|_{F}^{2}+\frac{2}{2 \theta+1} \Delta t \sum_{n=1}^{N-1} \nu\left\|\mathcal{J}_{n+\theta}^{\epsilon}\left(\nabla \boldsymbol{\xi}^{h}\right)\right\|^{2} \\
\leq & \exp \left(C \nu^{-1} T\right)\left[\left(\frac{2 \theta-1}{2 \theta+1}\right)^{N}\left\|\boldsymbol{\xi}_{0}^{h}\right\|^{2}+2\left(1-\left(\frac{2 \theta-1}{2 \theta+1}\right)^{N}\right)\left\|\left[\boldsymbol{\xi}_{1}^{h}\right]\right\|_{\boldsymbol{\xi}_{0}^{h}}^{2}\right] \|_{G} \\
& +C\left(1-\left(\frac{2 \theta-1}{2 \theta+1}\right)^{N}\right)\left(\nu^{-1}(\Delta t)^{4}\left\|\left|p_{t t}\right|\right\|_{2,0}^{2}+\nu^{-1} h^{2 s+2}\||p|\|_{2, s+1}^{2}\right. \\
& +\nu^{-1}(\Delta t)^{4}\left\|\left|\mathbf{u}_{t t t}\right|\right\|_{2,0}^{2}+\nu(\Delta t)^{4}\left\|\left|\nabla \mathbf{u}_{t t}\right|\right\|_{2,0}^{2}+\nu^{-1}(\Delta t)^{4}\left\|\left|\nabla \mathbf{u}_{t t}\right|\right\|_{2,0}^{2} \\
& +\nu^{-1}(\Delta t)^{4}\left\|\left|\nabla \mathbf{u}\left\|_{\infty, 0}^{2}\right\|\right| \nabla \mathbf{u}_{t t}\left|\left\|_{2,0}^{2}+\nu^{-1}(\Delta t)^{4}\right\| \nabla \mathbf{u}\left\|_{\infty, 0}^{2}\right\|\right| \nabla \mathbf{u}_{t t} \mid\right\|_{2,0}^{2} \\
& \left.\left.+\nu^{-1} h^{2 k+2}\left\|\left|\mathbf{u}_{t}\right|\right\|_{2, k+1}^{2}+\nu h^{2 k}\||\mathbf{u}|\|_{2, k+1}^{2}+\nu^{-1} h^{2 k}\||\nabla \mathbf{u}|\|_{\infty, 0}^{2}\||\mathbf{u}|\|_{2, k+1}^{2}\right)\right]
\end{aligned}
$$

Recall $\mathbf{e}_{n}=\boldsymbol{\eta}_{n}+\boldsymbol{\xi}_{n}^{h}$. By triangle inequality and Lemma 3.1, we obtain the error inequality (4.1).

5. Numerical Experiments. This section presents numerical experiments to test the family of proposed methods. Two typical methods, i.e., CNLEREg $\left(\theta=\frac{1}{2}\right)$ and BDFLE2REG $(\theta=1)$, are tested on several benchmark problems, including a problem with known analytical solution in order to verify predicted convergence rates, Poiseuille flow at low viscosity over a long time interval, flow past a normal flat plate, and channel flow with a contraction and two outlets. In all cases, the proposed methods perform very well.

For flow past a normal flat plate, we compare solutions for BDFLE2REG to another common stabilization method used with BDF2LE, which is discussed in the introduction as a 'speed' penalization. We will call it BDFLE2STAB, and it is given by

$$
\begin{aligned}
& \frac{3 \mathbf{u}_{n+1}-4 \mathbf{u}_{n}+\mathbf{u}_{n-1}}{2 \triangle t}-\nu \triangle \mathbf{u}_{n+1}-\alpha \triangle\left(\mathbf{u}_{n+1}-\mathbf{u}_{n}\right) \\
& \quad+\left(2 \mathbf{u}_{n}-\mathbf{u}_{n-1}\right) \cdot \nabla \mathbf{u}_{n+1}+\nabla p_{n+1}=\mathbf{f}_{n+1}, \\
& \nabla \cdot \mathbf{u}_{n+1}=0 .
\end{aligned}
$$

It will use a standard finite element spatial discretization in our simulations.

5.1. Convergence rate verification. Our first experiment tests the predicted convergence rates for the method. To do this, we selected the analytical solution

$$
\mathbf{u}=\left(\begin{array}{c}
\cos (y) e^{t} \\
\sin (x) e^{t}
\end{array}\right), p=(x-y)(1+t)
$$


took the domain to be the unit square, set the viscosity $\nu=1$, and then calculated the forcing $f$ from this data and the NSE. Errors and convergence rates were then calculated by varying the mesh width $h$ and timestep $\Delta t$, using $\left(P_{2}, P_{1}\right)$ Taylor-Hood elements on uniform meshes, and the CNLEREg algorithm. Full Dirichlet boundary conditions were enforced nodally on the boundary. For the spatial convergence rates, we fixed $\Delta t=T / 16$ and the end time $T=0.1$, and for the temporal convergence rates, we fixed the mesh width to be $h=1 / 128$ and set $T=2$.

Denoting

$$
\left\|\mathbf{u}-\mathbf{u}^{h}\right\|_{2,1}:=\left(\Delta t \sum_{n=1}^{N}\left\|\nabla\left(\mathbf{u}\left(t^{n}\right)-\mathbf{u}_{n}^{h}\right)\right\|^{2}\right)^{1 / 2},
$$

the results are shown in tables 5.1-5.2 (for spatial error and temporal error, respectively), for different choices of the stabilization parameter $\epsilon$. The spatial convergence rate appears to be 2 for each choice of $\epsilon$, which is optimal based on our element choice. The temporal convergence rate also appears to be 2 for each choice $\epsilon$, which is also optimal, although the actual error can be observed to increase as $\epsilon$ increases. Hence, these tables are consistent with the convergence rates predicted by the theory above, and thus provide some verification that the theory (and the code) are correct.

\begin{tabular}{|l|c|c|c|c|c|c|}
\hline & \multicolumn{2}{|c|}{$\epsilon=0$} & \multicolumn{2}{c|}{$\epsilon=10^{-2}$} & \multicolumn{2}{c|}{$\epsilon=1.0$} \\
\hline$h$ & $\left\|\mathbf{u}-\mathbf{u}^{h}\right\|_{2,1}$ & Rate & $\left\|\mathbf{u}-\mathbf{u}^{h}\right\|_{2,1}$ & Rate & $\left\|\mathbf{u}-\mathbf{u}^{h}\right\|_{2,1}$ & Rate \\
\hline$\frac{1}{4}$ & $7.5448 \mathrm{e}-4$ & & $7.5448 \mathrm{e}-4$ & & $7.5448 \mathrm{e}-4$ & \\
\hline$\frac{1}{8}$ & $1.8872 \mathrm{e}-4$ & 1.99924 & $1.8872 \mathrm{e}-4$ & 1.99924 & $1.8872 \mathrm{e}-4$ & 1.99926 \\
\hline$\frac{1}{16}$ & $4.7186 \mathrm{e}-5$ & 1.99982 & $4.7186 \mathrm{e}-5$ & 1.99982 & $4.7185 \mathrm{e}-5$ & 1.99982 \\
\hline$\frac{1}{32}$ & $1.1797 \mathrm{e}-5$ & 1.99997 & $1.1797 \mathrm{e}-5$ & 1.99996 & $1.1797 \mathrm{e}-5$ & 1.99989 \\
\hline$\frac{1}{64}$ & $2.9493 \mathrm{e}-6$ & 1.99995 & $2.9493 \mathrm{e}-6$ & 1.99995 & $2.9517 \mathrm{e}-6$ & 1.99886 \\
\hline$\frac{1}{128}$ & $7.3770 \mathrm{e}-7$ & 1.99927 & $7.3765 \mathrm{e}-7$ & 1.99936 & $7.4712 \mathrm{e}-7$ & 1.98212 \\
\hline
\end{tabular}

Table 5.1: This table gives errors and convergence rates for analytical test problem with $\nu=1.0$, $T=0.1, \Delta t=T / 16$, using CNLEReg and varying mesh widths.

\begin{tabular}{|l|c|c|c|c|c|r|}
\hline & \multicolumn{2}{|c|}{$\epsilon=0$} & \multicolumn{2}{c|}{$\epsilon=10^{-2}$} & \multicolumn{2}{c|}{$\epsilon=1.0$} \\
\hline$\Delta t$ & $\left\|\mathbf{u}-\mathbf{u}^{h}\right\|_{2,1}$ & Rate & $\left\|\mathbf{u}-\mathbf{u}^{h}\right\|_{2,1}$ & Rate & $\left\|\mathbf{u}-\mathbf{u}^{h}\right\|_{2,1}$ & Rate \\
\hline$\frac{T}{2}$ & $1.92362 \mathrm{e}-2$ & & $1.83172 \mathrm{e}-2$ & & $2.79061 \mathrm{e}-2$ & \\
\hline$\frac{T}{4}$ & $3.61969 \mathrm{e}-3$ & 2.40988 & $3.41510 \mathrm{e}-3$ & 2.42320 & $1.20402 \mathrm{e}-2$ & 1.21272 \\
\hline$\frac{T}{8}$ & $7.70911 \mathrm{e}-4$ & 2.23123 & $7.27397 \mathrm{e}-4$ & 2.23111 & $3.19789 \mathrm{e}-3$ & 1.91267 \\
\hline$\frac{T}{16}$ & $1.80583 \mathrm{e}-4$ & 2.09390 & $1.70331 \mathrm{e}-4$ & 2.09440 & $8.29912 \mathrm{e}-4$ & 1.94609 \\
\hline$\frac{T}{32}$ & $4.53413 \mathrm{e}-5$ & 1.99376 & $4.28931 \mathrm{e}-5$ & 1.98953 & $2.10445 \mathrm{e}-4$ & 1.97951 \\
\hline
\end{tabular}

Table 5.2: This table gives errors and convergence rates for analytical test problem with $\nu=1.0$, $T=2.0, h=1 / 128$, using CNLEReg and varying time step.

5.2. Poiseuille flow with small viscosity. Our second experiment tests the long-time stability of a Poiseuille channel flow simulation with viscosity $\nu=10^{-4}$. Although this type of flow is a smooth NSE solution, it is well known to have numerical instabilities when viscosity values are small. 
The computations use CNLEREG on the $[0,4] \times[0,1]$ rectangular domain, $\Delta t=$ $1.0, \mathbf{f}=\mathbf{0},\left(P_{2}, P_{1}\right)$ Taylor-Hood elements on a uniform coarse mesh with $h=0.1$, and an end time of $T=1,500$. We begin with an initial condition of

$$
\mathbf{u}_{0}=\left(\begin{array}{c}
4 y(1-y) \\
0
\end{array}\right)
$$

and enforce boundary conditions to be no-slip on the top and bottom of the channel, and parabolic (same as the initial condition) at the inlet and outlet. Solutions were then computed using $\epsilon=0$ (no stabilization) and with $\epsilon=10^{-5}, 10^{-4}$. Plots of energy $\frac{1}{2}\left\|\mathbf{u}_{h}^{n}\right\|^{2}$ versus time are shown in figure 5.1 for the three solutions, and reveals that while the stabilized solutions maintain the same (correct) energy throughout the simulation, at around $T=800$ the unstabilized solution undergoes a transition away from the correct solution. After $T=800$, the energy in the unstabilized system becomes incorrect and then oscillates in time. Plots of the velocity field speed contours for the $\epsilon=0$ and $10^{-4}$ solutions at $\mathrm{T}=1,500$ are shown in figure 5.2, and from the plots we observe that the stabilized solution has maintained its smooth parabolic profile, while the unstabilized solution has clearly diverged from the correct solution it started with.

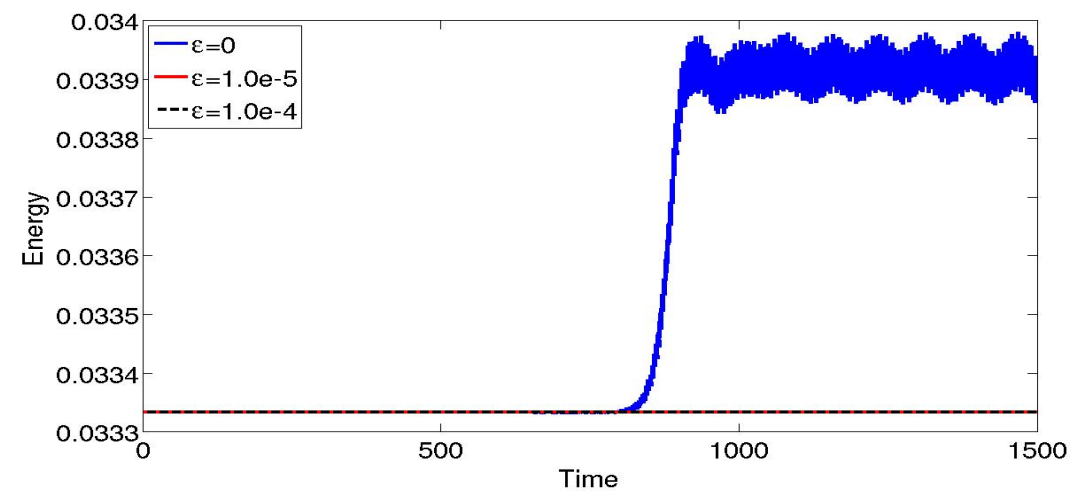

Fig. 5.1: $\frac{1}{2}\|\mathbf{u}\|^{2}$ vs. Time plots with $\nu=10^{-4}, T=1500, h=0.2, \Delta t=1$ for different values of $\epsilon$.
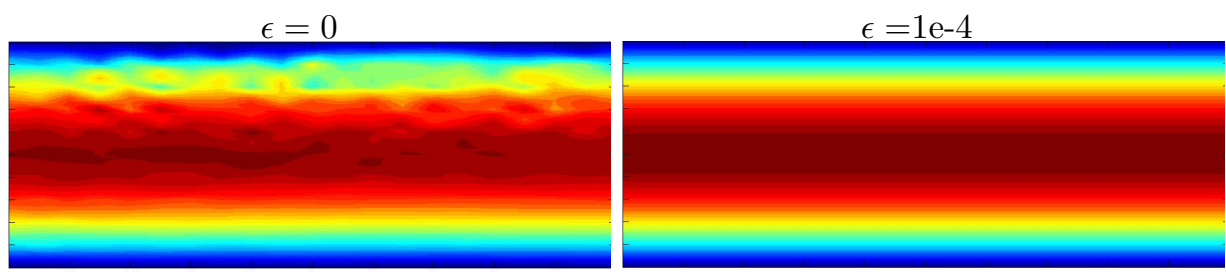

Fig. 5.2: CNLEREg computed velocity field speed contours for Poiseuille flow at $T=1500$, with $\epsilon=0$ (left) and $\epsilon=0.0001$ (right).

5.3. Flow past a normal flat plate. Next, we consider simulating flow past a normal flat plate with the proposed scheme BDF2LEREG, following the experiments 
from $[29,30]$. We use the $[-7,20] \times[-10,10]$ rectangular channel as the domain, with a $0.125 \times 1$ flat plate vertically centered and placed 7 units into the channel from the left. The inflow velocity is set with $\mathbf{u}_{i n}=\langle 1,0\rangle^{T}$, no forcing is applied $(\mathbf{f}=\mathbf{0})$, and the kinematic viscosity is taken to be $\nu=0.01$, which gives a Reynolds number of $R e=100$ (based on the plate height). No-slip conditions are enforced on the walls and plate, and for the outflow, the zero-traction boundary condition is enforced weakly via the 'do-nothing' condition. The quantities of interest are the Strouhal number St (the frequency of vortex shedding) and the time averaged drag coefficient, both of which are computed from the final 10 periods of the flow after the flow reaches a statistically steady (periodic-in-time) state (which occurs at around $\mathrm{T}=130$ ). The Strouhal number is calculated as in [29, 30], using the fast Fourier transform (FFT) of the transverse velocity at $(4.0,0.0)$ (note since the maximum inlet speed is 1 and fluid density is assumed to be 1 , we get directly that $S t$ is the dominant frequency of the FFT). The drag coefficients are computed at each timestep and then averaged, using a global integral formula (see [19] for details).

The computations used $\left(P_{2}, P_{1}\right)$ Taylor-Hood elements for velocity and pressure, on a Delaunay-generated triangulation which provided 16,013 total degrees of freedom (dof). For these tests we used $\theta=1$ (i.e. BDF2LEREG), varied $\Delta t=0.05,0.1$, and 0.25 , and set the regularization parameter $\epsilon=0.002$. For comparison, we also computed using no stabilization with the same discretizations, and also no stabilization on a much finer discretization with that used $159 \mathrm{~K}$ total dof and $\Delta t=0.01$. We also compare our results with data from direct numerical simulations of [29, 30]. The goal here is to show that when large time step sizes are used, the proposed stabilization can provide significantly better solutions.

Comparisons between the solutions are given in table 5.3 and figure 5.3. Table 5.3 shows the time averaged drag coefficients and Strouhal numbers for the simulations, as well as for DNS data. We first observe that our solution on a fine mesh with a small timestep matches the DNS of Saha in both the Strouhal number and the time averaged drag coefficient. On the coarser discretizations, the stabilized solutions are significantly more accurate than the unstabilized solutions, particularly as the timestep size increases. In particular, the Strouhal numbers for the stabilized solutions match the DNS data (up to the spacing of discrete frequencies from the FFT), and the drag coefficients are reasonably accurate, even for $\Delta t=0.25$. Note that the curvature stabilization of BDF2LEREG has a decreasing affect on the drag coefficient, which is in sharp contrast to the results below for BDF2LESTAB (where speed is stabilized).

In figure 5.3, we plot the speed contours (zoomed in near the plate) of the fine mesh solution, and stabilized and unstabilized coarse mesh solutions with $\Delta t=0.25$, at a snapshot in time, $T=200$. We observe that the stabilized coarse mesh solution qualitatively matches the fine mesh solution much better than the unstabilized solution. That is, the stabilized solution has the same flow structures in nearly the same locations as the fine mesh solution, while the unstabilized solution appears quite different. Flow structures of the unstabilized solution not matching the fine mesh solution are consistent with the significant error in Strouhal number of the unstabilized solution.

We also compare results for BDF2LEREg to those of BDF2LESTAB. Hence on the same coarse spatial discretization, we compute solutions with BDF2LESTAB with $\Delta t=0.1$ and 0.25 , and varying $\alpha$. Time averaged drag coefficients and Strouhal numbers for each simulation are given in table 5.4. We observe that the BDF2LEREG solutions provides significantly better drag coefficients compared to BDF2LESTAB 
Fine mesh

$\Delta t=0.01, \epsilon=0$

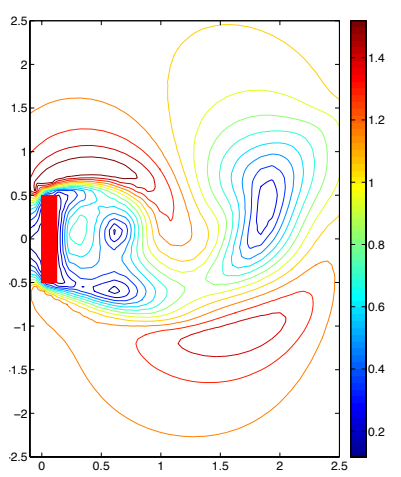

Coarse mesh

$\Delta t=0.25, \epsilon=0$

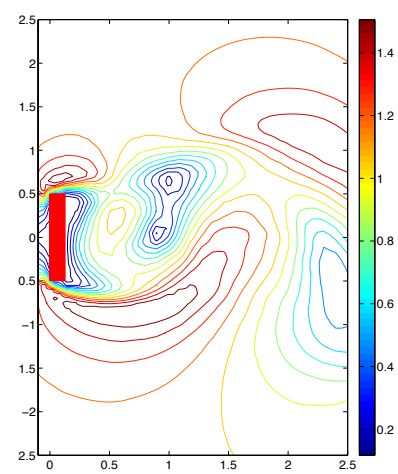

Coarse mesh $\Delta t=0.25, \epsilon=0.002$

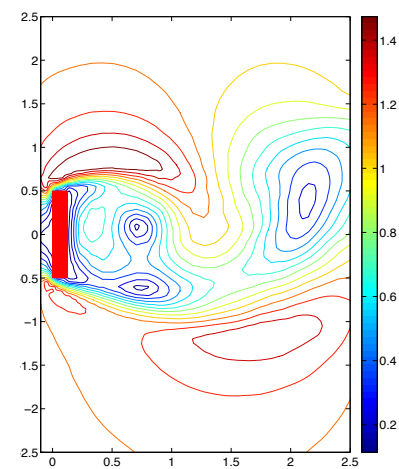

Fig. 5.3: Shown above is a (zoomed) contour plot of the speed at time $\mathrm{T}=200$ for a resolved solution (left), and unstabilized (center) and stabilized (right) coarse discretization solutions.

\begin{tabular}{|c|c|c|c|c|c|}
\hline Method & mesh & $\Delta t$ & $\epsilon$ & $C_{d}$ & Strouhal number \\
\hline DNS of Saha [29, 30] & fine & 0.0001 & - & 2.60 & 0.183 \\
\hline & & & & & \\
\hline BDF2LE (no stab) & fine & 0.01 & 0 & 2.59 & 0.183 \\
\hline & & & & & \\
\hline BDF2LE (no stab) & coarse & 0.05 & 0 & 2.64 & $0.186^{*}$ \\
\hline BDF2LEREG & coarse & 0.05 & 0.002 & 2.61 & $0.186^{*}$ \\
\hline & & & & & \\
\hline BDF2LE (no stab) & coarse & 0.1 & 0 & 2.68 & 0.195 \\
\hline BDF2LEREG & coarse & 0.1 & 0.002 & 2.58 & $0.186^{*}$ \\
\hline & & & & & \\
\hline BDF2LE (no stab) & coarse & 0.25 & 0 & 3.05 & 0.211 \\
\hline BDF2LEREG & coarse & 0.25 & 0.002 & 2.53 & $0.180^{*}$ \\
\hline
\end{tabular}

Table 5.3: Shown above are long-time average drag coefficients and Strouhal numbers for the simulations with and without stabilization, along with DNS data from finer discretizations. We note that the Strouhal numbers with asterisks are the closest discrete frequency values in the FFT to 0.183 .

(for any parameter $\alpha$ ). It is not unexpected that BDF2LESTAB does a poor job with drag coefficient prediction, since it stabilizes speed; in fact it is clear in the table that increasing $\alpha$ increases the drag coefficient.

For $\Delta t=0.1$, BDF2LESTAB does accurately predict the Strouhal number, even though $C_{d}$ prediction is inaccurate and gets worse as the stabilization parameter is increased. For $\Delta t=0.25$, however, the BDF2LESTAB solutions do not do a good job with Strouhal number prediction. Only for $\alpha=0.1$ does it accurately predict the Strouhal number, but this particular solution has a terrible prediction of $C_{d}$.

5.4. Channel flow with two outlets and a contraction. For our final numerical test, we apply the proposed method to a 2D benchmark contracted channel flow problem, first studied by Turek et. al. in [15], with one inlet on the left hand 


\begin{tabular}{|c|c|c|c|c|c|}
\hline Method & mesh & $\Delta t$ & $\alpha$ & $C_{d}$ & Strouhal number \\
\hline BDF2LE (no stab) & coarse & 0.1 & 0 & 2.68 & 0.195 \\
\hline BDF2LESTAB & coarse & 0.1 & 0.005 & 2.68 & $0.186^{*}$ \\
\hline BDF2LESTAB & coarse & 0.1 & 0.01 & 2.69 & $0.186^{*}$ \\
\hline BDF2LESTAB & coarse & 0.1 & 0.05 & 2.79 & $0.186^{*}$ \\
\hline BDF2LESTAB & coarse & 0.1 & 0.1 & 2.89 & $0.186^{*}$ \\
\hline & & & & & \\
\hline BDF2LE (no stab) & coarse & 0.25 & 0 & 3.05 & 0.211 \\
\hline BDF2LESTAB & coarse & 0.25 & 0.005 & 3.07 & 0.203 \\
\hline BDF2LESTAB & coarse & 0.25 & 0.01 & 3.08 & 0.203 \\
\hline BDF2LESTAB & coarse & 0.25 & 0.05 & 3.21 & 0.195 \\
\hline BDF2LESTAB & coarse & 0.25 & 0.1 & 3.38 & $0.180^{*}$ \\
\hline
\end{tabular}

Table 5.4: Shown above are long-time average drag coefficients and Strouhal numbers for the simulations with BDF2LESTAB, with varying $\alpha$ and $\Delta t$, using the same 18,822 dof mesh as BDF2LEREG uses above. We note that the Strouhal numbers with asterisks are the closest discrete frequency values in the FFT to 0.183 .

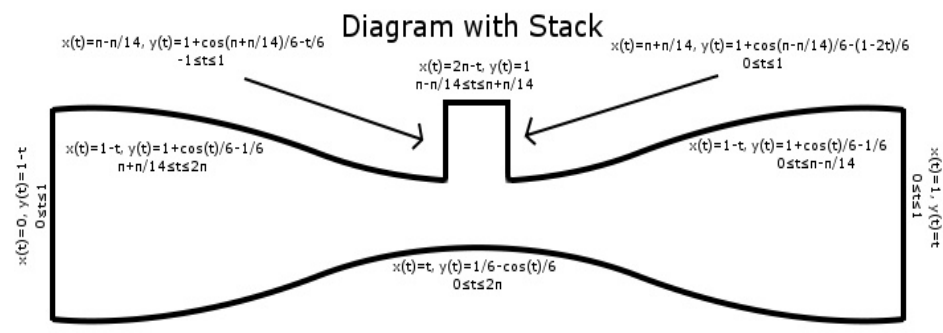

Fig. 5.4: The physical model of the domain for contracted channel flow with two outlets is shown above.

side, one vertical outlet in the middle and one outlet on the right hand side, as shown in Figure 5.4. We enforce a parabolic inflow profile $\mathbf{u}=\langle 4 y(1-y), 0\rangle^{T}$ with maximum horizontal velocity component $\mathbf{u}_{\text {inlet }}^{\max }=1$ at the center, no slip boundary conditions on its sides, and at the outflows, zero traction is imposed (with the 'do-nothing' condition). We impose no external forcing $(\mathbf{f}=\mathbf{0})$, set the kinematic viscosity $\nu=0.001$, start the flow from rest, and the simulation is run to $T=4$.

A resolved solution of the NSE was found using a timestep of $\Delta t=0.005$ and 260,378 total spatial dof in [3], and its $\mathrm{T}=4$ speed contours are shown in figure 5.5.

We computed using $\left(P_{2}, P_{1}\right)$ Taylor-Hood elements on a coarse mesh that provided 18,822 total dof, using time step size $\Delta t=0.02$ with both BDF2 without stabilization (i.e. BDF2LEReg with $\epsilon=0$ ), and BDF2LEReg with stabilization parameter $\epsilon=$ 0.0005 . Speed contours of these solutions at $\mathrm{T}=4$ are shown in figure 5.5 , and we observe that the unstabilized solution is destroyed by oscillations. The stabilized solution, however, remains stable and is in general qualitative agreement with the DNS, although there are some differences near the end of the channel; however on such a coarse discretization, it is not expected to get exact agreement. 

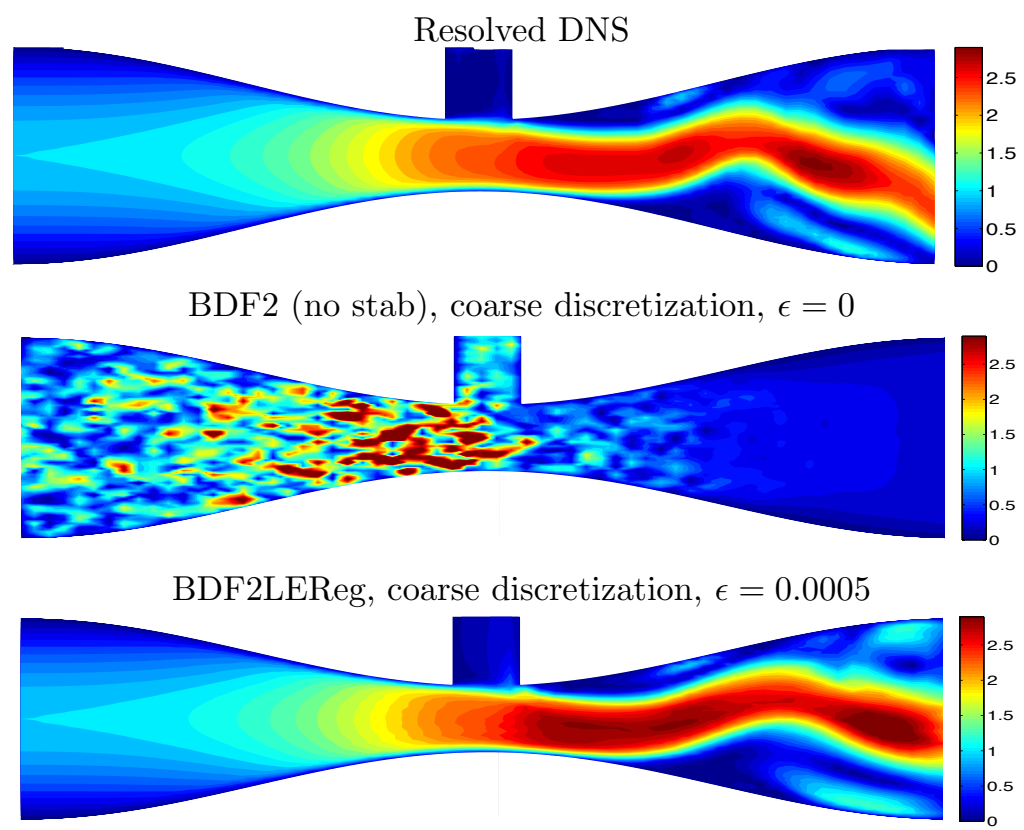

Fig. 5.5: Shown above is contour plot of the speed of the resolved Navier-Stokes velocity solution at $\mathrm{T}=4$, along with stabilized and unstabilized coarse discretization solutions.

6. Conclusions. A family of efficient, stabilized, second order, unconditionally stable timestepping methods for NSE are analyzed and numerically tested. These methods require the solution of only one linear system per time step, and have enhanced stability due to a penalization of the velocity curvature, and achieve second order temporal accuracy because the penalization is itself second order consistent. Rigorous proofs of unconditional stability and convergence are given for the entire family of methods.

In addition to the theoretical results, several numerical experiments are performed which show the effectiveness of the methods. In addition to verifying the theoreticallypredicted convergence rates, a significant positive effect of the method is seen on the stability and accuracy of solutions of three benchmark flow problems, when compared to solutions of unstabilized schemes and common, related, stabilized schemes. In particular, the method BDF2LEREG is shown to provide significantly more accurate predictions of the drag coefficient for flow across a flat plate, compared to BDF2LESTAB.

The methods proposed herein can be easily applied to many other models with minor modifications, and this report is an initial attempt to study these linearized methods and new stabilization in a CFD context. How the stabilization terms affect the stability separately still requires more investigation. Generally speaking, more extensive numerical experiments are needed, and in particular for problems where there exists intermittency and large temporal variation.

\section{REFERENCES}

[1] F. Armero And J. C. Simo, Long-term dissipativity of time-stepping algorithms for an ab- 
stract evolution equation with applications to the incompressible MHD and Navier-Stokes equations, Comput. Methods Appl. Mech. Engrg., 131 (1996), pp. 41-90. 1

[2] G. BAKER, Galerkin approximations for the Navier-Stokes equations, tech. rep., Harvard University, 1976. 1

[3] A. Bowers and L. Rebholz, Numerical study of a regularization model for incompressible flow with deconvolution-based adaptive nonlinear filtering, Computer Methods in Applied Mechanics and Engineering, 258 (2013), pp. 1-12. 18

[4] S. C. Brenner And L. R. Scott, The mathematical theory of finite element methods, vol. 15 of Texts in Applied Mathematics, Springer-Verlag, New York, 1994. 4

[5] K. BRYAN, Accelerating the convergence to equilibrium of ocean-climate models, Journal of Physical Oceanography, 14 (1984), pp. 666-673. 2

[6] Y. CaO, E. Lunasin, And E. Titi, Global well-posedness of the three-dimensional viscous and inviscid simplified Bardina turbulence models, Commun. Math. Sci., 4 (2006), pp. 823-848. 2

[7] W. Chen, M. Gunzburger, D. Sun, And X. Wang, Efficient and long-time accurate secondorder methods for the Stokes-Darcy system, SIAM J. Numer. Anal., 51 (2013), pp. 25632584. 2

[8] L. Davis And F. Pahlevani, Semi-implicit schemes for transient Navier-Stokes equations and eddy viscosity models, Numerical Methods for Partial Differential Equations, 25 (2009), pp. $212-231.2$

[9] V. Girault and P.-A. Raviart, Finite element approximation of the Navier-Stokes equations, vol. 749 of Lecture Notes in Mathematics, Springer-Verlag, Berlin, 1979. 4, 13

[10] V. Girault and P.-A. Raviart, Finite element methods for Navier-Stokes equations : theory and algorithms, Springer-Verlag, 1986. 11

[11] M. Gunzburger, E. Lee, Y. Saka, C. Trenchea, and X. Wang, Analysis of nonlinear spectral eddy-viscosity models of turbulence, J. Sci. Comput., 45 (2010), pp. 294-332. 3

[12] M. D. Gunzburger, Finite element methods for viscous incompressible flows, Computer Science and Scientific Computing, Academic Press Inc., Boston, MA, 1989. A guide to theory, practice, and algorithms. 4

[13] E. Hairer And G. Wanner, Solving ordinary differential equations. II, vol. 14 of Springer Series in Computational Mathematics, Springer-Verlag, Berlin, 2010. Stiff and differentialalgebraic problems, Second revised edition. 5

[14] Y. HE, Two-level method based on finite element and Crank-Nicolson extrapolation for the timedependent Navier-Stokes equations, SIAM J. Numer. Anal., 41 (2003), pp. 1263-1285. 1, 7

[15] J. Heywood, R. Rannacher, and S. Turek, Artificial boundaries and flux and pressure conditions for the incompressible Navier-Stokes equations, International Journal for Numerical Methods in Fluids, 22 (1996), pp. 325-352. 17

[16] J. G. HeYwood AND R. RANNACHER, Finite-element approximation of the nonstationary Navier-Stokes problem. IV. Error analysis for second-order time discretization, SIAM J. Numer. Anal., 27 (1990), pp. 353-384. 7, 11

[17] T. Hughes, A. A. Oberai, And L. Mazzei, Large Eddy Simulation of Turbulent Channel Flow by the Variational Multiscale Method, Phys. Fluids, 13 (2001), pp. 1784-1799. 2

[18] N. Hurl, W. Layton, Y. Li, And C. Trenchea, Stability analysis of the Crank-NicolsonLeapfrog method with the Robert-Asselin-Williams time filter, BIT Numerical Mathematics, (2014), pp. 1-13. 2

[19] V. John, Large eddy simulation of turbulent incompressible flows, vol. 34 of Lecture Notes in Computational Science and Engineering, Springer-Verlag, Berlin, 2004. Analytical and numerical results for a class of LES models. 16

[20] V. John And S. KAYA, A finite element variational multiscale method for the Navier-Stokes equations, SIAM J. Sci. Comp., 26 (2005), pp. 1485 - 1503. 2

[21] H. Johnston And J.-G. Liu, Accurate, stable and efficient Navier-Stokes solvers based on explicit treatment of the pressure term, J. Comput. Phys., 199 (2004), pp. 221-259. 3

[22] P. Kuberry, A. Larios, L. Rebholz, and N. Wilson, Numerical approximation of the Voigt regularization for incompressible Navier-Stokes and magnetohydrodynamic flows, Computers and Mathematics with Applications, 64 (2012), pp. 2647-2662. 2

[23] A. Labovsky, W.Layton, C. Manica, M. Neda, and L. Rebholz, The Stabilized Extrapolated Trapezoidal Finite-Element Method for the Navier-Stokes Equations, Comput. Methods. Appl. Mech. Engrg., 198 (2009), pp. 958-974. 2, 5

[24] O. A. LADYŽEnSKAJA, Modifications of the Navier-Stokes equations for large gradients of the velocities, Zap. Naučn. Sem. Leningrad. Otdel. Mat. Inst. Steklov. (LOMI), 7 (1968), pp. 126154. 3 
[25] W. LAYTON, Introduction to the numerical analysis of incompressible viscous flows, vol. 6 of Computational Science \& Engineering, Society for Industrial and Applied Mathematics (SIAM), Philadelphia, PA, 2008. With a foreword by Max Gunzburger. 11

[26] W. Layton, Y. Li, And C. Trenchea, Recent developments in IMEX methods with time filters for systems of evolution equations, J. Comput. Appl. Math., 299 (2016), pp. 50-67. 2

[27] Y. Li and C. Trenchea, A higher-order Robert-Asselin type time filter, J. Comput. Phys., 259 (2014), pp. 23-32. 2

[28] J.-L. Lions, Quelques méthodes de résolution des problèmes aux limites non linéaires, Dunod, 1969. 3

[29] A. SAHA, Far-wake characteristics of two-dimensional flow past a normal flat plate, Physics of Fluids, 19 (2007), pp. 128110:1-4. 16, 17

[30] S. SAHA, Direct numerical simulation of two-dimensional flow past a normal flat plate, Journal of Engineering Mechanics, 139 (2013), pp. 1894-1901. 16, 17

[31] J. C. Simo, F. Armero, ANd C. A. Taylor, Stable and time-dissipative finite element methods for the incompressible Navier-Stokes equations in advection dominated flows, Internat. J. Numer. Methods Engrg., 38 (1995), pp. 1475-1506. 1

[32] C. TREncheA, Second order implicit for local effects and explicit for nonlocal effects is unconditionally stable, tech. rep., University of Pittsburgh, 2012. 2, 5

[33] — Stability of partitioned IMEX methods for systems of evolution equations with skewsymmetric coupling, ROMAI J., 10 (2014), pp. 175-189. 2

[34] X. WANG, An efficient second order in time scheme for approximating long time statistical properties of the two dimensional Navier-Stokes equations, Numer. Math., 121 (2012), pp. $753-779.5$

[35] P. D. Williams, A Proposed Modification to the Robert-Asselin Time Filter, Mon. Wea. Rev., 137 (2009), pp. 2538-2546. 2 